\title{
NONCOMMUTATIVE JOINT DILATIONS AND FREE PRODUCT OPERATOR ALGEBRAS
}

\author{
Gelu Popescu
}

Let $\mathcal{A}_{n}(n=2,3, \ldots$, or $n=\infty)$ be the noncommutative disc algebra, and $\mathcal{O}_{n}$ (resp. $\mathcal{T}_{n}$ ) be the Cuntz (resp. Toeplitz) algebra on $n$ generators. Minimal joint isometric dilations for families of contractive sequences of operators on a Hilbert space are obtained and used to extend the von Neumann inequality and the commutant lifting theorem to our noncommutative setting.

We show that the universal algebra generated by $k$ contractive sequences of operators and the identity is the amalgamated free product operator algebra $\tilde{*}_{\mathrm{C}} \mathcal{A}_{n_{i}}$ for some positive integers $n_{1}, n_{2}, \ldots, n_{k} \geq 1$, and characterize the completely bounded representations of $\tilde{*}_{\mathrm{C}} \mathcal{A}_{n_{i}}$. It is also shown that ${ }_{{ }^{C}} \mathcal{A}_{n_{i}}$ is completely isometrically imbedded in the "biggest" free product $C^{*}$-algebra $\check{*}_{C} \mathcal{T}_{n_{i}}$ (resp. $\left.\tilde{*}_{C} \mathcal{O}_{n_{i}}\right)$, and that all these algebras are completely isometrically isomorphic to some universal free operator algebras, providing in this way some factorization theorems.

We show that the free product disc algebra $\check{*}_{\mathrm{C}} \mathcal{A}_{n_{i}}$ is not amenable and the set of all its characters is homeomorphic to $\overline{\left(\mathrm{C}^{n_{1}}\right)_{1}} \times \cdots \times \overline{\left(\mathrm{C}^{n_{k}}\right)_{1}}$.

An extension of the Naimark dilation theorem to free semigroups is considered. This is used to construct a large class of positive definite operator-valued kernels on the unital free semigroup on $n$ generators and to study the class $\mathcal{C}_{\rho}(\rho>0)$ of $\rho$-contractive sequences of operators.

The dilation theorems are also used to extend the operatorial trigonometric moment problem to the free product $C^{*}$-algebras $\check{*}_{\mathrm{C}} \mathcal{T}_{n_{i}}$ and $\check{*}_{\mathrm{C}} \mathcal{O}_{n_{i}}$.

\section{Introduction and preliminaries.}

Let $\mathcal{H}$ be a complex Hilbert space and $B(\mathcal{H})$ the set of bounded linear operators on $\mathcal{H}$. We identify $M_{m}(B(\mathcal{H}))$, the set of $m \times m$ matrices with entries from $B(\mathcal{H})$, with $B(\underbrace{\mathcal{H} \oplus \cdots \oplus \mathcal{H}}_{\text {m-times }})$. Thus we have a natural $C^{*}$-norm on $M_{m}(B(\mathcal{H}))$. If $X$ is an operator space, i.e., a linear subspace of $B(\mathcal{H})$, we 
consider $M_{m}(X)$ as a subspace of $M_{m}(B(\mathcal{H}))$ with the induced norm. The appropriate morphisms between operator spaces are the completely bounded maps $[\mathbf{A r}],[\mathbf{P 2}],[\mathbf{P i}]$. Let $X, Y$ be operator spaces and $u: X \rightarrow Y$ be a linear map. Define $u_{m}: M_{m}(X) \rightarrow M_{m}(Y)$ by

$$
u_{m}\left(\left[x_{i j}\right]\right)=\left[u\left(x_{i j}\right)\right] .
$$

We say that $u$ is completely bounded ( $c b$ in short) if

$$
\|u\|_{c b}=\sup _{m \geq 1}\left\|u_{m}\right\|<\infty .
$$

If $\|u\|_{c b} \leq 1$ (resp. $u_{m}$ is an isometry for any $m \geq 1$ ) then $u$ is completely contractive (resp. isometric), and if $u_{m}$ is positive for all $m$, then $u$ is called completely positive.

Let $n=2,3, \ldots$, and $v_{1}, v_{2}, \ldots, v_{n}$ be isometries satisfying

$$
v_{i}^{*} v_{j}=\delta_{i j} 1 \text {. }
$$

We proved in [Po4] that $\operatorname{Alg}\left(1, v_{1}, \ldots, v_{n}\right)$, the closed non-selfadjoint algebra generated by $1, v_{1}, \ldots, v_{n}$, is completely isometrically isomorphic to the noncommutative disc algebra $\mathcal{A}_{n}[\mathbf{P o 2}]$. Let us recall $[\mathbf{C u}]$ that the Cuntz algebra $\mathcal{O}_{n}$ (resp. Toeplitz algebra $\mathcal{T}_{n}$ ) is uniquely defined as the $C^{*}$ algebra generated by $n$ isometries satisfying (1.1) and $\sum_{i=1}^{n} v_{i} v_{i}^{*}=1$ (resp. $\sum_{i=1}^{n} v_{i} v_{i}^{*}<1$ ). If $n=\infty$, only the condition (1.1) is required to define $\mathcal{A}_{\infty}, \mathcal{O}_{\infty}$, and $\mathcal{T}_{\infty}$. If $n=1$, we set $\mathcal{A}_{1}:=A(\mathbf{D})$, the classical disc algebra, $\mathcal{O}_{1}:=C(\mathbf{T})$, and $\mathcal{T}_{1}:=C^{*}(S)$, the $C^{*}$-algebra generated by the unilateral shift $S$ acting on the Hardy space $H^{2}(\mathbf{T})$ (see $[\mathbf{C}]$ ). Since $\mathcal{A}_{n}$ is completely isometrically imbedded in $\mathcal{O}_{n}$ (resp. $\mathcal{T}_{n}$ ) one can view the noncommutative disc algebra as a "non-selfadjoint Cuntz algebra" as well as an "analytic Toeplitz algebra". Let us mention that $\mathcal{A}_{n}$ is the universal algebra generated by a row contraction and the identity [Po6], and if $n \neq m$ then $\mathcal{A}_{n}$ is not Banach isomorphic to $\mathcal{A}_{m}$ [Po4]. For a concrete realization of $\mathcal{A}_{n}$ and $\mathcal{T}_{n}$ see $[\mathbf{P o} 2],[\mathbf{P o} 4]$.

In Section 2 we obtain a minimal joint isometric dilation for any sequence of contractive sequences of operators on a Hilbert space, extending in this way the Schaffer's construction $[\mathbf{S c}],[\mathbf{S z F} 2]$ and also some results from $[\mathbf{F}]$, $[\mathbf{B u}],[\mathbf{P o 1}],[\mathbf{D S z}]$. Other dilation theorems are obtained using some results from $[\mathbf{A r}],[\mathbf{B o}],[\mathbf{S}],[\mathbf{P o 1}],[\mathbf{P o 2}],[\mathbf{P o 3}]$, and $[\mathbf{P o} 4]$. Some consequences of these joint isometric dilations are considered in this paper.

The free product of $C^{*}$-algebras has been studied by many authors ([Av $]$, $[\mathbf{B o}],[\mathbf{B P}],[\mathbf{V}]$, etc.) but still remains misterious. We consider here the so-called "biggest" free product of operator algebras $[\mathbf{B P}]$.

Inequalities of von Neumann type are considered in Section 2 and Section 3, extending some results from [vN], [A], [Boz], [Po2], [Po3], [Po4], [Po7]. Let $k \geq 1$ and $n_{1}, \ldots, n_{k} \geq 1$ be fixed positive integers, and $\left\{x_{i j}\right\} \begin{gathered}\substack{i=1,2, \ldots, k \\ j=1,2, \ldots, n_{i}} \\ \text {, }\end{gathered}$ 
$\left\{y_{i j}\right\}_{\substack{i=1,2, \ldots, k \\ j=1,2, \ldots, n_{i}}}$ be noncommuting indeterminates satisfying the relation

$$
y_{i \alpha} x_{i \beta}=\delta_{\alpha \beta} 1, \text { for any } i=1,2, \ldots, k \text {, and } \alpha, \beta=1,2, \ldots, n_{i} \text {. }
$$

Let $\mathcal{P}$ be the set of all reduced polynomials in these indeterminates, i.e., each monomial is in reduced form according to the relation (1.2). Let $\left(T_{i 1}, \ldots, T_{i n_{i}}\right), i=1,2, \ldots, k$, be contractive sequences of operators on a Hilbert space $\mathcal{H}$, i.e.,

$$
T_{i 1} T_{i 1}^{*}+\cdots+T_{i n_{i}} T_{i n_{i}}^{*} \leq I_{\mathcal{H}}, \quad i=1,2, \ldots, k .
$$

For each polynomial $p=p\left(1,\left\{x_{i j}\right\},\left\{y_{i j}\right\}\right) \in \mathcal{P}$ let us define the operator $p\left(I_{\mathcal{H}},\left\{T_{i j}\right\},\left\{T_{i j}^{*}\right\}\right)$ acting on the Hilbert space $\mathcal{H}$. We prove in Section 3 the following extension of the von Neumann inequality

$$
\left\|p\left(I_{\mathcal{H}},\left\{T_{i j}\right\},\left\{T_{i j}^{*}\right\}\right)\right\| \leq\|p\|_{\tilde{*}_{\mathbf{C}}} \mathcal{T}_{n_{i}},
$$

where $p \in \mathcal{P}$ is viewed as an element in the free product $C^{*}$-algebra $\widetilde{*}_{\mathbf{C}} \mathcal{T}_{n_{i}}$ (see Section 3 ). On the other hand, for any polynomial $q\left(1,\left\{x_{i j}\right\}\right)$ in noncommuting indeterminates $\left\{x_{i j}\right\}_{\substack{i=1,2, \ldots, k \\ j=1,2, \ldots, n_{i}}}$, we prove that

$$
\left\|q\left(I_{\mathcal{H}},\left\{T_{i j}\right\}\right)\right\| \leq\|q\|_{\check{*}_{\mathbf{C}} \mathcal{O}_{n_{i}}}=\|q\|_{\ddot{*}_{\mathbf{C}} \mathcal{T}_{n_{i}}} \leq\|q\|_{\check{*}_{\mathbf{C}} C\left(\mathbf{T}_{r}\right)},
$$

where $\mathbf{T}_{r}=\mathbf{T}$, the circle group, $r=1,2, \ldots, n_{1}+n_{2}+\cdots+n_{k}$, and $q\left(1,\left\{x_{i j}\right\}\right)$ is seen as an element of $\check{*}_{\mathbf{C}} \mathcal{O}_{n_{i}}, \check{*}_{\mathbf{C}} \mathcal{T}_{n_{i}}$, and $\check{*}_{\mathbf{C}} C\left(\mathbf{T}_{r}\right)$, respectively. If a sequence of operators $\left\{T_{i j}\right\}_{\substack{i=1,2, \ldots, k \\ j=1,2, \ldots, n_{i}}} \subset B(\mathcal{H})$ satisfies the relation

$$
T_{i 1} T_{i 1}^{*}+\cdots+T_{i n_{i}} T_{i n_{i}}^{*}=I_{\mathcal{H}}, \quad i=1,2, \ldots, k,
$$

then the inequality (1.4) is extended to

$$
\left\|q\left(I_{\mathcal{H}},\left\{T_{i j}\right\},\left\{T_{i j}^{*}\right\}\right)\right\| \leq\|q\|_{\widetilde{*}_{\mathbf{C}} \mathcal{O}_{n_{i}}} \leq\|q\|_{\check{F}_{\mathbf{C}} \mathcal{T}_{n_{i}}},
$$

for any polynomial $p\left(1,\left\{x_{i j}\right\},\left\{y_{i j}\right\}\right) \in \mathcal{P}$.

Let $\mathcal{A}_{n_{i}}(i=1,2, \ldots, k)$ be the noncommutative disc algebra and $\check{*}_{\mathbf{C}} \mathcal{A}_{n_{i}}$ be the amalgamated (over the identity) free product operator algebra. We prove in Section 3 that the universal algebra generated by $k$ contractive sequences of operators and the identity is the free product disc algebra $\check{*}_{\mathbf{C}} \mathcal{A}_{n_{i}}$, for some integers $n_{1}, \ldots, n_{k} \geq 1$. Moreover, using Paulsen's result [P1], we give a complete characterization of the completely bounded (resp. contractive) representations of $\check{*}_{\mathbf{C}} \mathcal{A}_{n_{i}}$. We shall prove that $\check{*}_{\mathbf{C}} \mathcal{A}_{n_{i}}$ is completely isometrically imbedded in $\check{*}_{\mathbf{C}} \mathcal{T}_{n_{i}}$ (resp. $\tilde{*}_{\mathbf{C}} \mathcal{O}_{n_{i}}$ ). On the other hand, it is proved that all these algebras are completely isometrically isomorphic to some free operator algebras of type $O A(\Delta, \mathcal{R})$, considered by Blecher $[\mathbf{B}]$. This identification together with the internal characterization of the matrix norm on a universal algebra $[\mathbf{B}],[\mathbf{B P}]$ lead to factorization theorems of type considered in $[\mathbf{B}],[\mathbf{B P}],[\mathbf{P o 6}]$.

In Section 4 we shall show that the set of all characters (multiplicative functionals) on $\mathcal{A}_{n_{1}} \check{*}_{\mathbf{C}} \cdots \check{*}_{\mathbf{C}} \mathcal{A}_{n_{k}}$ is homeomorphic to $\overline{\left(\mathbf{C}^{n_{1}}\right)_{1}} \times \cdots \times \overline{\left(\mathbf{C}^{n_{k}}\right)_{1}}$ 
and that the first cohomology group of $\mathcal{A}_{n_{1}} \check{*}_{\mathbf{C}} \cdots \check{*}_{\mathbf{C}} \mathcal{A}_{n_{k}}$ with coefficients in $\mathbf{C}$ is isomorphic to the additive group $\mathbf{C}^{n_{1}+\cdots+n_{k}}$. In particular, this shows that the free product disc algebra is not amenable.

In Section 5 we consider an extension of the Naimark dilation theorem $[\mathbf{N}]$ to free semigroups and construct a large class of positive definite operatorvalued kernels on the unital free semigroup on $n$ generators. As an application, we define the class $\mathcal{C}_{\rho}(\rho>0)$ of $\rho$-contractive sequences of operators and prove, using the results from the preceeding sections, that any sequence of class $\mathcal{C}_{\rho}$ is simultaneously similar to a sequence of class $\mathcal{C}_{1}$, extending in this way the classical result of Sz.-Nagy and Foiaş [SzF1] (see also [Po5]).

In Section 6, using some joint dilation theorems from Section 1, we extend the operatorial trigonometric moment problem $[\mathbf{A k}],[\mathbf{P o 5}]$ to the free product $C^{*}$-algebras $\check{*}_{\mathbf{C}} \mathcal{T}_{n_{i}}$ and $\check{*}_{\mathbf{C}} \mathcal{O}_{n_{i}}$.

Let us remark that in the particular case when $n_{1}=n_{2}=\cdots=n_{k}=n$ one can obtain joint dilations and universal algebras associated to $k \times n$ operator matrices $\left[T_{i j}\right]$ with contractive rows. On the other hand, let us mention that if $k, n_{1}, \ldots, n_{k}$ are infinite all the results of this paper hold true in a slightly adapted version.

\section{Joint minimal isometric dilations.}

Let $k \geq 1$ and $n_{1}, \ldots, n_{k} \geq 1$ be fixed positive integers. For each $i=$ $1,2, \ldots, k$, let $\left(T_{i 1}, \ldots, T_{i n_{i}}\right)$ be a contractive sequence of operators on a Hilbert space $\mathcal{H}$, i.e.,

$$
T_{i 1} T_{i 1}^{*}+\cdots+T_{i n_{i}} T_{i n_{i}}^{*} \leq I_{\mathcal{H}}
$$

In what follows we extend the noncommutative dilation theorem [Po1] to our setting. The following result also subsumes the isometric dilation theorems from $[\mathbf{S z F} 2],[\mathbf{F}],[\mathbf{B u}]$, and $[\mathbf{D S z}]$.

Theorem 2.1. Let $\left(T_{i 1}, \ldots, T_{i n_{i}}\right), i=1,2, \ldots, k$, be contractive sequences of operators on a Hilbert space $\mathcal{H}$. Then there exists a Hilbert space $\mathcal{K} \supset \mathcal{H}$ and contractive sequences $\left(V_{i 1}, \ldots, V_{i n_{i}}\right), i=1,2, \ldots, k$, of isometries on $\mathcal{K}$ with the following properties:

(i) $\left.V_{i j}^{*}\right|_{\mathcal{H}}=T_{i j}^{*} \quad\left(i=1,2, \ldots, k, j=1,2, \ldots, n_{i}\right)$;

(ii) $P_{\mathcal{K} \ominus \mathcal{H}} V_{i_{1} j_{1}} \mathcal{K} \perp P_{\mathcal{K} \ominus \mathcal{H}} V_{i_{2} j_{2}} \mathcal{K}$ if $i_{1} \neq i_{2}, j_{1}=1,2, \ldots, n_{i_{1}}$, and $j_{2}=$ $1,2, \ldots, n_{i_{2}}$

(iii) $\mathcal{K}=\mathcal{H} \bigvee V_{i_{1} j_{1}} \cdots V_{i_{p} j_{p}} \mathcal{H} \quad$ (any finite product in $V_{i j}$ is considered).

Moreover, the joint isometric dilation satisfying these properties is uniquely determined up to an isomorphism.

Proof. For each $i=1,2, \ldots, k$, let us consider the operator matrix $T_{i}:=$ $\left[T_{i 1} \ldots T_{i n_{i}}\right]$ and let $D_{T_{i}}$ be the defect operator defined on $\oplus_{j=1}^{n_{i}} \mathcal{H}$ by $D_{T_{i}}=$ 
$\left(I-T_{i}^{*} T_{i}\right)^{1 / 2}$. Let

$$
\mathcal{D}=\mathcal{D}_{1} \oplus \mathcal{D}_{2} \oplus \cdots \oplus \mathcal{D}_{k}
$$

where $\mathcal{D}_{i}=\overline{D_{T_{i}}\left(\oplus_{j=1}^{n_{i}} \mathcal{H}\right)}$. Let $n=n_{1}+n_{2}+\cdots+n_{k}$ and consider the full Fock space $[\mathbf{E}]$

$$
F^{2}\left(H_{n}\right)=\mathbf{C} 1 \oplus \oplus_{m \geq 1} H_{n}^{\otimes m},
$$

where $H_{n}$ is an $n$-dimensional complex Hilbert space with orthonormal basis $\left\{e_{i j}\right\}_{\substack{i=1,2, \ldots, k \\ j=1,2, \ldots, n_{i}}}$. For each $i=1,2, \ldots, k$ and $j=1,2, \ldots, n_{i}$, let $S_{i j} \in$ $B\left(F^{2}\left(H_{n}\right)\right)$ be the left creation operator with $e_{i j}$, i.e.,

$$
S_{i j} \xi=e_{i j} \otimes \xi, \xi \in F^{2}\left(H_{n}\right) .
$$

Consider the operator $D_{i j}: \mathcal{H} \rightarrow F^{2}\left(H_{n}\right) \otimes \mathcal{D}$ defined by

$$
\begin{aligned}
& D_{i j} h \\
& =1 \otimes(\underbrace{0 \oplus \cdots \oplus 0}_{i-1 \text { times }} \oplus D_{T_{i}}(\underbrace{0, \ldots, 0}_{j-1 \text { times }}, h, \underbrace{0, \ldots, 0}_{n_{i}-j \text { times }}) \oplus \underbrace{0 \oplus \cdots \oplus 0}_{k-i \text { times }}) \oplus 0 \oplus 0 \\
& \quad+\ldots
\end{aligned}
$$

for any $h \in \mathcal{H}$. Consider the Hilbert space

$$
\mathcal{K}=\mathcal{H} \oplus\left(F^{2}\left(H_{n}\right) \otimes \mathcal{D}\right) .
$$

For each $i=1,2, \ldots, k$ and $j=1,2, \ldots, n_{i}$, we define the operator $V_{i j}$ on $\mathcal{K}$ by

$$
V_{i j}(h \oplus(\xi \otimes d))=T_{i j} h \oplus\left(D_{i j} h+\left(S_{i j} \otimes I_{\mathcal{D}}\right)(\xi \otimes d)\right),
$$

for any $h \in \mathcal{H}, \xi \in F^{2}\left(H_{n}\right)$, and $d \in \mathcal{D}$. One can see that

$$
V_{i j}=\left[\begin{array}{cc}
T_{i j} & 0 \\
D_{i j} & S_{i j} \otimes I_{\mathcal{D}}
\end{array}\right]
$$

with respect to the decomposition (2.1). It follows that

$$
V_{i j}^{*} V_{i j}=\left[\begin{array}{cc}
T_{i j}^{*} T_{i j}+D_{i j}^{*} D_{i j} & D_{i j}^{*}\left(S_{i j} \otimes I_{\mathcal{D}}\right) \\
\left(S_{i j}^{*} \otimes I_{\mathcal{D}}\right) D_{i j} & S_{i j}^{*} S_{i j} \otimes I_{\mathcal{D}}
\end{array}\right] .
$$

Using the definition of $D_{i j}$, an easy computation shows that $T_{i j}^{*} T_{i j}+D_{i j}^{*} D_{i j}=$ $I_{\mathcal{H}}$ and $\left(S_{i j}^{*} \otimes I_{\mathcal{D}}\right) D_{i j}=0$. Since $S_{i j}^{*} S_{i j}=I$, it follows that $V_{i j}^{*} V_{i j}=I_{\mathcal{K}}$. According to the relation $(2.2)$, it is clear that $\left.V_{i j}^{*}\right|_{\mathcal{H}}=T_{i j}^{*}$. If $i=1,2, \ldots, k$ is fixed and $\alpha, \beta=1,2, \ldots, n_{i}, \alpha \neq \beta$, then one can similarily prove that 
$V_{i \alpha}^{*} V_{i \beta}=0$. This shows that $\left(V_{i 1}, \ldots, V_{i n_{i}}\right)$ is a contractive sequence of isometries. On the other hand, we have

$$
P_{\mathcal{K} \ominus \mathcal{H}}^{\mathcal{K}} V_{i j}=\left[\begin{array}{cc}
0 & 0 \\
D_{i j} & S_{i j} \otimes I_{\mathcal{D}}
\end{array}\right] .
$$

According to the definition of the operators $D_{i j}$, and since $\left\{S_{i j}\right\}$ are isometries with orthogonal ranges, one can infer that

$$
P_{\mathcal{K} \ominus \mathcal{H}} V_{i_{1} j_{1}} \mathcal{K} \perp P_{\mathcal{K} \ominus \mathcal{H}} V_{i_{2} j_{2}} \mathcal{K}
$$

if $i_{1} \neq i_{2}, j_{1}=1,2, \ldots, n_{i_{1}}, j_{2}=1,2, \ldots, n_{i_{2}}$.

Let us verify that $\left\{V_{i j}\right\}_{\substack{i=1,2, \ldots, k \\ j=1,2, \ldots, n_{i}}}$ is the minimal isometric dilation of $\left\{T_{i j}\right\}_{\substack{i=1,2, \ldots, k \\ j=1,2, \ldots, n_{i}}}$. Consider $\mathcal{H}_{1}:=\mathcal{H} \bigvee\left(\bigvee_{\substack{i=1,2, \ldots, k \\ j=1,2, \ldots, n_{i}}} V_{i j} \mathcal{H}\right)$ and

$$
\mathcal{H}_{q}:=\mathcal{H}_{q-1} \bigvee\left(\bigvee_{\substack{i=1,2, \ldots, k \\ j=1,2, \ldots, n_{i}}} V_{i j} \mathcal{H}_{q-1}\right), \quad \text { if } q \geq 2
$$

It is easy to see that $\mathcal{H}_{1}=\mathcal{H} \oplus(\mathbf{C} 1 \otimes \mathcal{D})$ and

$$
\mathcal{H}_{q}=\mathcal{H} \oplus\left(\mathbf{C} 1 \oplus \oplus_{m=1}^{q-1} H_{n}^{\otimes m}\right) \otimes \mathcal{D}, \quad \text { if } q \geq 2 .
$$

Clearly we have $\mathcal{H}_{q} \subset \mathcal{H}_{q+1}$ and

$$
\bigvee_{q=1}^{\infty} \mathcal{H}_{q}=\mathcal{H} \oplus\left(F^{2}\left(H_{n}\right) \otimes \mathcal{D}\right) .
$$

Hence, and according to (2.1), we infer that

$$
\mathcal{K}=\mathcal{H} \bigvee V_{i_{1} j_{1}} \cdots V_{i_{p} j_{p}} \mathcal{H}
$$

Let us show that the minimal isometric dilation $\left\{V_{i j}\right\}$ of $\left\{T_{i j}\right\}$ is unique up to a unitary operator. Following the classical case, it is enough to show that the inner product

$$
L:=\left\langle V_{i_{1} j_{1}} \cdots V_{i_{p} j_{p}} h, V_{\alpha_{1} \beta_{1}} \cdots V_{\alpha_{q} \beta_{q}} h^{\prime}\right\rangle,
$$

$\left(h, h^{\prime} \in \mathcal{H}\right)$, depends only on the operators $T_{i j}(i=1,2, \ldots, k ; j=1,2, \ldots$, $\left.n_{i}\right)$. We can assume that $\left(i_{1}, j_{1}\right) \neq\left(\alpha_{1}, \beta_{1}\right)$. If $i_{1}=\alpha_{1}$ and $j_{1} \neq \beta_{1}$ then $V_{\alpha_{1} \beta_{1}}^{*} V_{i_{1} j_{1}}=0$, hence $L=0$. If $i_{1} \neq \alpha_{1}$ then $P_{\mathcal{K} \ominus \mathcal{H}} V_{i_{1} j_{1}} \mathcal{K} \perp P_{\mathcal{K} \ominus \mathcal{H}} V_{\alpha_{1} \beta_{1}} \mathcal{K}$. Therefore,

$$
\begin{aligned}
L & =\left\langle\left(P_{\mathcal{H}}+P_{\mathcal{K} \ominus \mathcal{H}}\right) V_{i_{1} j_{1}} \cdots V_{i_{p} j_{p}} h,\left(P_{\mathcal{H}}+P_{\mathcal{K} \ominus \mathcal{H}}\right) V_{\alpha_{1} \beta_{1}} \cdots V_{\alpha_{q} \beta_{q}} h^{\prime}\right\rangle \\
& =\left\langle P_{\mathcal{H}} V_{i_{1} j_{1}} \cdots V_{i_{1} j_{p}} h, P_{\mathcal{H}} V_{\alpha_{1}, \beta_{1}} \cdots V_{\alpha_{q} \beta_{q}} h^{\prime}\right\rangle \\
& =\left\langle T_{i_{1} j_{1}} \cdots T_{i_{p} j_{p}} h, T_{\alpha_{1} \beta_{1}} \cdots T_{\alpha_{q} \beta_{q}} h^{\prime}\right\rangle .
\end{aligned}
$$


Let $\left\{V_{i j}^{\prime}\right\}_{\substack{i=1,2, \ldots, k \\ j=1,2, \ldots, n_{i}}}$ be another minimal isometric dilation of $\left\{T_{i j}\right\}_{\substack{i=1,2, \ldots, k \\ j=1,2, \ldots, n_{i}}}^{\substack{i \\ n^{\prime}}}$ on a Hilbert space $\mathcal{K}^{\prime} \supset \mathcal{H}$. Setting

$$
U\left(\sum_{\text {finite }} V_{i_{1} j_{1}} \cdots V_{i_{p} j_{p}} h_{i_{1} j_{1}, \ldots, i_{p} j_{p}}\right)=\sum_{\text {finite }} V_{i_{1} j_{1}}^{\prime} \cdots V_{i_{p} j_{p}}^{\prime} h_{i_{1} j_{1}, \ldots, i_{p} j_{p}}
$$

with $h_{i_{1} j_{1}, \ldots, i_{p} j_{p}} \in \mathcal{H}$, we define an isometric operator. Since the isometric dilations are minimal, the operator $U$ can be extended by continuity to a unitary from $\mathcal{K}$ to $\mathcal{K}^{\prime}$. The proof is complete.

Let $\left\{x_{i j}\right\}_{\substack{i=1,2, \ldots, k \\ j=1,2, \ldots, n_{i}}}$ and $\left\{y_{i j}\right\}_{\substack{i=1,2, \ldots, k \\ j=1,2, \ldots, n_{i}}}$ be noncommuting indeterminates satisfying the relation

$$
y_{i \alpha} x_{i \beta}=\delta_{\alpha \beta} 1, \text { for any } i=1,2, \ldots, k \text {, and } \alpha, \beta=1,2, \ldots, n_{i} \text {. }
$$

Let $\mathcal{P}$ be the set of all reduced polynomials in these indeterminates, i.e., each monomial is in reduced form according to the above mentioned relation.

The following version of von Neumann's inequality $[\mathbf{v N}]$ holds.

Corollary 2.2. For every polynomial $p\left(1,\left\{x_{i j}\right\},\left\{y_{i j}\right\}\right) \in \mathcal{P}$ and $\left\{T_{i j}\right\}_{\substack{i=1,2, \ldots, k \\ j=1,2, \ldots, n_{i}}}$ $\subset \mathcal{B}(\mathcal{H})$ such that

$$
T_{i 1} T_{i 1}^{*}+\cdots+T_{i n_{i}} T_{i n_{i}}^{*} \leq I_{\mathcal{H}}, \quad i=1,2, \ldots, k,
$$

we have

$$
\left\|p\left(I_{\mathcal{H}},\left\{T_{i j}\right\},\left\{T_{i j}^{*}\right\}\right)\right\| \leq \sup \left\|p\left(I,\left\{V_{i j}\right\},\left\{V_{i j}^{*}\right\}\right)\right\|,
$$

where the supremum is taken over all contractive sequences of isometries $\left(V_{i 1}, \ldots, V_{i n_{i}}\right)(i=1,2, \ldots, k)$ on a Hilbert space.

Proof. Let $\left\{V_{i j}\right\}_{\substack{i=1,2, \ldots, k \\ j=1,2, \ldots, n_{i}}} \subset \mathcal{B}(\mathcal{K})$ be the minimal joint isometric dilation of $\left\{T_{i j}\right\}_{\substack{i=1,2, \ldots, k \\ j=1,2, \ldots, n_{i}}}$ in the sense of Theorem 2.1. Using the properties of this dilation, one can prove that

$$
\left.P_{\mathcal{H}} V_{i_{1} j_{1}} \cdots V_{i_{p} j_{p}} V_{r_{1} q_{1}}^{*} \cdots V_{r_{m} q_{m}}^{*}\right|_{\mathcal{H}}=T_{i_{1} j_{1}} \cdots T_{i_{p} j_{p}} T_{r_{1} q_{1}}^{*} \cdots T_{r_{m} q_{m}}^{*}
$$

and

$$
\left.P_{\mathcal{H}} V_{i_{1} j_{1}}^{*} \cdots V_{i_{p} j_{p}}^{*} V_{r_{1} q_{1}} \cdots V_{r_{m} q_{m}}\right|_{\mathcal{H}}=T_{i_{1} j_{1}}^{*} \cdots T_{i_{p} j_{p}}^{*} T_{r_{1} q_{1}} \cdots T_{r_{m} q_{m}}
$$

if $i_{p} \neq r_{1}$. Now, using these relations, one can see that, for any polynomial $p\left(1,\left\{x_{i j}\right\},\left\{y_{i j}\right\}\right) \in \mathcal{P}$,

$$
\left.P_{\mathcal{H}} p\left(I_{\mathcal{K}},\left\{V_{i j}\right\},\left\{V_{i j}^{*}\right\}\right)\right|_{\mathcal{H}}=p\left(I_{\mathcal{H}},\left\{T_{i j}\right\},\left\{T_{i j}^{*}\right\}\right) .
$$

Hence, we deduce (2.3). This completes the proof.

We can apply [Ar, Theorem 1.3.1] to our setting in order to get the following commutant lifting theorem for $C^{*}\left(\left\{T_{i j}\right\}\right)^{\prime}$. 
Theorem 2.3. Let $\left\{T_{i j}\right\}_{\substack{i=1,2, \ldots, k \\ j=1,2, \ldots, n_{i}}} \subset \mathcal{B}(\mathcal{H})$ be such that $\left(T_{i 1}, \ldots, T_{i n_{i}}\right)$ is contractive for each $i=1,2, \ldots, k$, and let $\left\{V_{i j}\right\}_{\substack{i=1,2, \ldots, k \\ j=1,2, \ldots, n_{i}}} \subset \mathcal{B}(\mathcal{K})$ be its minimal isometric dilation. If $X \in C^{*}\left(\left\{T_{i j}\right\}\right)^{\prime}$ then there is a unique $\tilde{X} \in$ $C^{*}\left(\left\{V_{i j}\right\}\right)^{\prime} \cap\left\{P_{\mathcal{H}}\right\}^{\prime}$ such that $\left.P_{\mathcal{H}} \tilde{X}\right|_{\mathcal{H}}=X$, where $P_{\mathcal{H}}$ is the orthogonal projection from $\mathcal{K}$ onto $\mathcal{H}$. Moreover, the map $X \rightarrow \tilde{X}$ is a $*$-isomorphism.

A particular case which can be proved directly is the following. The proof is similar to [BrJ, Lemma 6.2], so we omit it.

Corollary 2.4. If $U \in C^{*}\left(\left\{T_{i j}\right\}\right)^{\prime}$ is a unitary then it has a unitary extension $\tilde{U} \in C^{*}\left(\left\{V_{i j}\right\}\right)^{\prime}$. Moreover this extension is unique.

Let $\mathcal{A}, \mathcal{B}$ be unital $C^{*}$-algebras and let $\mathcal{A} *_{\mathbf{C}} \mathcal{B}$ be their algebraic free product amalgamated over the identity, which is a $*$-algebra. For $x \in \mathcal{A} *_{\mathrm{C}} \mathcal{B}$ define

$$
\|x\|=\sup \{\|\pi(x)\|\}
$$

where the supremum is taken over all $*$-representations of $\mathcal{A} *_{\mathbf{C}} \mathcal{B}$. Let us mentioned that all $*$-representations of $\mathcal{A} *_{\mathbf{C}} \mathcal{B}$ are in one-to-one correspondence with pairs of $*$-representations of $\mathcal{A}$ and $\mathcal{B}$, which act on the same Hilbert space. The "biggest" free product of $\mathcal{A}$ and $\mathcal{B}$ is the completion of $\mathcal{A} *_{\mathbf{C}} \mathcal{B}$ in this norm, and is denoted by $\mathcal{A} \check{*}_{\mathbf{C}} \mathcal{B}$ (see $[\mathbf{A v}]$ ).

Theorem 2.5. For each $i=1,2, \ldots, k$, let $\left(T_{i 1}, \ldots, T_{i n_{i}}\right)$ be a contractive sequence of operators on a Hilbert space $\mathcal{H}$. Then there exists a Hilbert space $\mathcal{K} \supset \mathcal{H}$ and contractive sequences $\left(V_{i 1}, \ldots, V_{i n_{i}}\right)(i=1,2, \ldots, k)$ of isometries on $\mathcal{K}$ such that

$$
V_{i 1} V_{i 1}^{*}+\cdots+V_{i n_{i}} V_{i n_{i}}^{*}=I_{\mathcal{K}} \quad(i=1,2, \ldots, k)
$$

and

$$
p\left(I_{\mathcal{H}},\left\{T_{i j}\right\}\right)=\left.P_{\mathcal{H}} p\left(I_{\mathcal{K}},\left\{V_{i j}\right\}\right)\right|_{\mathcal{H}}
$$

for any polynomial $p$ in noncommuting indeterminates $\left\{x_{i j}\right\}_{\substack{i=1,2, \ldots, k \\ j=1,2, \ldots, n_{i}}}$.

Proof. Consider the case $k \geq 2$. For each $i=1,2, \ldots, k$, let $\sigma_{i 1}, \ldots, \sigma_{i n_{i}}$ be a system of generators for the Cuntz algebra $\mathcal{O}_{n_{i}}$. We proved in [Po4] that the Banach algebras $\operatorname{Alg}\left(1, \sigma_{i 1}, \ldots, \sigma_{i n_{i}}\right)$ and the noncommutative disc algebra $\mathcal{A}_{n_{i}}$ are completely isometrically isomorphic. According to the noncommutative von Neumann inequality [Po2], [Po4], we infer that the map

$$
\Phi_{i}: \operatorname{Alg}\left(1, \sigma_{i 1}, \ldots, \sigma_{i n_{i}}\right) \rightarrow B(\mathcal{H})
$$

defined by

$$
\Phi\left(p\left(1, \sigma_{i 1}, \ldots, \sigma_{i n_{i}}\right)\right)=p\left(I_{\mathcal{H}}, T_{i 1}, \ldots, T_{i n_{i}}\right)
$$

is a completely contractive homomorphism.

Using the extension theorem of Arveson $[\mathbf{A r}]$ we infer that there is a completely positive linear map $\Psi_{i}: \mathcal{O}_{n_{i}} \rightarrow B(\mathcal{H})$ such that $\left.\Psi_{i}\right|_{A l g\left(1, \sigma_{i 1}, \ldots, \sigma_{i n_{i}}\right)}=$ 
$\Phi_{i}$. Now using Boca's result [Bo], there is a common completely positive extension

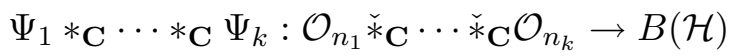

with

$$
\left(\Psi_{1} *_{\mathbf{C}} \cdots *_{\mathbf{C}} \Psi_{k}\right)\left(p\left(1,\left\{\sigma_{i j}\right\}\right)\right)=p\left(I_{\mathcal{H}},\left\{T_{i j}\right\}\right),
$$

where $p\left(1,\left\{\sigma_{i j}\right\}\right) \in \mathcal{O}_{n_{1}} \check{*}_{\mathbf{C}} \cdots \check{*}_{\mathbf{C}} \mathcal{O}_{n_{k}}$ is any polynomial in $1, \sigma_{i 1}, \ldots, \sigma_{i n_{i}}$ $\left(i=1,2, \ldots, k ; j=1,2, \ldots, n_{i}\right)$. According to Stinespring's theorem $[\mathbf{S}]$,

$$
\left(\Psi_{1} *_{\mathbf{C}} \cdots *_{\mathbf{C}} \Psi_{k}\right)\left(p\left(1,\left\{\sigma_{i j}\right\}\right)\right)=\left.P_{\mathcal{H}} \pi\left(p\left(1,\left\{\sigma_{i j}\right\}\right)\right)\right|_{\mathcal{H}}
$$

for any $p\left(1,\left\{\sigma_{i j}\right\}\right) \in \mathcal{O}_{n_{1}} \check{*}_{\mathbf{C}} \cdots \check{*}_{\mathbf{C}} \mathcal{O}_{n_{k}}$, where $\pi$ is a $*$-representation of $\mathcal{O}_{n_{1}} \tilde{*}_{\mathbf{C}} \cdots \tilde{*}_{\mathbf{C}} \mathcal{O}_{n_{k}}$ on a Hilbert space $\mathcal{K} \supset \mathcal{H}$, and $P_{\mathcal{H}}$ is the orthogonal projection of $\mathcal{K}$ onto $\mathcal{H}$. In particular, we have

$$
p\left(I_{\mathcal{H}},\left\{T_{i j}\right\}\right)=\left.P_{\mathcal{H}} p\left(I_{\mathcal{K}},\left\{\pi\left(\sigma_{i j}\right)\right\}\right)\right|_{\mathcal{H}} .
$$

Notice that $\left(\pi\left(\sigma_{i 1}\right), \ldots, \pi\left(\sigma_{i n_{i}}\right)\right)$ is a sequence of isometries such that

$$
\pi\left(\sigma_{i 1}\right) \pi\left(\sigma_{i 1}\right)^{*}+\cdots+\pi\left(\sigma_{i n_{i}}\right) \pi\left(\sigma_{i n_{i}}\right)^{*}=I_{\mathcal{K}}, i=1,2, \ldots, k .
$$

Denote $V_{i j}=\pi\left(\sigma_{i j}\right), \quad i=1,2, \ldots, k ; j=1,2, \ldots, n_{i}$. This completes the proof when $k \geq 2$. The case $k=1$ can be treated similarly (see also $[\mathrm{Bo}])$.

Let $\mathcal{P}_{x}$ be the set of all polynomials in noncommuting indeterminates $x_{i j}$ $\left(i=1,2, \ldots, k ; j=1,2, \ldots, n_{i}\right)$. Notice that $\mathcal{P}_{x} \subset \mathcal{P}$.

Corollary 2.6. For every polynomial $p\left(1,\left\{x_{i j}\right\}\right) \in \mathcal{P}_{x}$ and $\left\{T_{i j}\right\}_{\substack{i=1,2, \ldots, k \\ j=1,2, \ldots, n_{i}}} \subset$ $B(\mathcal{H})$ such that $\left(T_{i 1}, \ldots, T_{i n_{i}}\right)$ is contractive for any $i=1,2, \ldots, k$,

$$
\left\|p\left(I_{\mathcal{H}},\left\{T_{i j}\right\}\right)\right\| \leq \sup \left\|p\left(I_{\mathcal{K}},\left\{V_{i j}\right\}\right)\right\|
$$

where the supremum is taken over all sequences of isometries $\left(V_{i 1}, \ldots, V_{i n_{i}}\right)$ $(i=1,2, \ldots, k)$ on a Hilbert space $\mathcal{K}$ such that

$$
V_{i 1} V_{i 1}^{*}+\cdots+V_{i n_{i}} V_{i n_{i}}^{*}=I_{\mathcal{K}}, \quad i=1,2, \ldots, k .
$$

Let us remark that in the particular case when $n_{1}=n_{2}=\cdots=n_{k}=1$ one obtains Bozejko's version [Boz] of von Neumann's inequality [vN $]$. On the other hand, in the particular case when $k=1, n_{1}=n$ we find a version of the noncommutative von Neumann inequality obtained in [Po2].

Theorem 2.7. Let $\left(T_{i 1}, \ldots, T_{i n_{i}}\right), i=1,2, \ldots, k$, be contractive sequences of operators on a Hilbert space $\mathcal{H}$ such that

$$
T_{i 1} T_{i 1}^{*}+\cdots+T_{i n_{i}} T_{i n_{i}}^{*}=I_{\mathcal{H}}, \quad i=1,2, \ldots, k .
$$

Then there exists a Hilbert space $\mathcal{K} \supset \mathcal{H}$ and contractive sequences $\left(V_{i 1}, \ldots\right.$, $\left.V_{i n_{i}}\right), i=1,2, \ldots, k$, of isometries on $\mathcal{K}$ with the following properties:

(i) $V_{i 1} V_{i 1}^{*}+\cdots+V_{i n_{i}} V_{i n_{i}}^{*}=I_{\mathcal{K}} \quad(i=1,2, \ldots k)$;

(ii) $\left.V_{i j}^{*}\right|_{\mathcal{H}}=T_{i j}^{*} \quad\left(i=1,2, \ldots, k, j=1,2, \ldots, n_{i}\right)$; 
(iii) For any polynomial $p\left(1,\left\{x_{i j}\right\},\left\{y_{i j}\right\}\right) \in \mathcal{P}$,

$$
\left.P_{\mathcal{H}} p\left(I_{\mathcal{K}},\left\{V_{i j}\right\},\left\{V_{i j}^{*}\right\}\right)\right|_{\mathcal{H}}=p\left(I_{\mathcal{H}},\left\{T_{i j}\right\},\left\{T_{i j}^{*}\right\}\right) ;
$$

(iv) $\mathcal{K}=\mathcal{H} \bigvee V_{i_{1} j_{1}} \cdots V_{i_{p} j_{p}} \mathcal{H}$ (any finite product in $V_{i j}$ is considered). Moreover, the joint isometric dilation satisfying these properties is uniquely determined up to an isomorphism.

Proof. Let $k \geq 2$ and $i \in\{1,2, \ldots, k\}$ be fixed. Since (2.5) holds, according to $\left[\mathbf{P o 1}\right.$, Proposition 2.5], there is a Hilbert space $\mathcal{K}_{i} \supset \mathcal{H}$ and a contractive sequence $\left(W_{i 1}, \ldots, W_{i n_{i}}\right)$ of isometries on $\mathcal{K}_{i}$ having the following properties:

(i) $W_{i 1} W_{i 1}^{*}+\cdots+W_{i n_{i}} W_{i n_{i}}^{*}=I_{\mathcal{K}_{i}}$;

(ii) $\left.W_{i j}^{*}\right|_{\mathcal{H}}=T_{i j}^{*} \quad\left(j=1,2, \ldots, n_{i}\right)$.

Therefore, for any polynomial $p\left(1,\left\{x_{i j}\right\},\left\{y_{i j}\right\}\right) \in \mathcal{P}$, we have

$$
\left.P_{\mathcal{H}} p\left(I_{\mathcal{K}_{i}},\left\{W_{i j}\right\},\left\{W_{i j}^{*}\right\}\right)\right|_{\mathcal{H}}=p\left(I_{\mathcal{H}},\left\{T_{i j}\right\},\left\{T_{i j}^{*}\right\}\right) .
$$

For each $i=1,2, \ldots, k$, let $\sigma_{i 1}, \ldots, \sigma_{i n_{i}}$ be a system of generators of the Cuntz algebra $\mathcal{O}_{n_{i}}$. Since the Cuntz algebra does not depend on the generators $[\mathbf{C u}]$, and using (2.6), we infer that the map $\phi_{i}: \mathcal{O}_{n_{i}} \rightarrow B(\mathcal{H})$ defined by

$$
\phi_{i}\left(p\left(1,\left\{\sigma_{i j}\right\},\left\{\sigma_{i j}^{*}\right\}\right)\right)=p\left(I_{\mathcal{H}},\left\{T_{i j}\right\},\left\{T_{i j}^{*}\right\}\right)
$$

is completely contractive, hence completely positive. Using Boca's result $[\mathbf{B o}]$, there is a common completely positive extension

$$
\Psi_{1} *_{\mathbf{C}} \cdots *_{\mathbf{C}} \Psi_{k}: \mathcal{O}_{n_{1}} \check{*}_{\mathbf{C}} \cdots \check{*}_{\mathbf{C}} \mathcal{O}_{n_{k}} \rightarrow B(\mathcal{H})
$$

with

$$
\left(\Psi_{1} *_{\mathbf{C}} \cdots *_{\mathbf{C}} \Psi_{k}\right)\left(p\left(1,\left\{\sigma_{i j}\right\},\left\{\sigma_{i j}^{*}\right\}\right)\right)=p\left(I_{\mathcal{H}},\left\{T_{i j}\right\},\left\{T_{i j}^{*}\right\}\right),
$$

for any $p\left(1,\left\{x_{i j}\right\},\left\{y_{i j}\right\}\right) \in \mathcal{P}$. According to Stinespring's theorem $[\mathbf{S}]$,

$$
\left(\Psi_{1} *_{\mathbf{C}} \cdots *_{\mathbf{C}} \Psi_{k}\right)\left(p\left(1,\left\{\sigma_{i j}\right\},\left\{\sigma_{i j}^{*}\right\}\right)\right)=\left.P_{\mathcal{H}} p\left(I_{\mathcal{K}},\left\{\pi\left(\sigma_{i j}\right)\right\},\left\{\pi\left(\sigma_{i j}^{*}\right)\right\}\right)\right|_{\mathcal{H}},
$$

where $\pi$ is a $*$-representation of $\mathcal{O}_{n_{1}} \check{*}_{\mathbf{C}} \cdots \check{*}_{\mathbf{C}} \mathcal{O}_{n_{k}}$ on a Hilbert space $\mathcal{K} \supset \mathcal{H}$, and $P_{\mathcal{H}}$ is the orthogonal projection of $\mathcal{K}$ onto $\mathcal{H}$. Denote $V_{i j}=\pi\left(\sigma_{i j}\right)$ $\left(i=1,2, \ldots, k ; j=1,2, \ldots, n_{i}\right)$. Now, it is easy to see that $\left\{V_{i j}\right\}$ satisfies all the properties stated in the theorem. Let us just mention that the property (ii) follows from the relation

$$
\left.P_{\mathcal{H}} V_{i j} V_{i j}^{*}\right|_{\mathcal{H}}=\left(\left.P_{\mathcal{H}} V_{i j}\right|_{\mathcal{H}}\right)\left(\left.P_{\mathcal{H}} V_{i j}^{*}\right|_{\mathcal{H}}\right),
$$

using an argument from $[\mathbf{A}]$.

The uniqueness is a consequence of Stinespring's theorem. Notice that the case $k=1$ can be treated similarly. This completes the proof.

Let us remark that, in the setting of Theorem 2.7, one can obtain a commutant lifting theorem similar to Theorem 2.3. 
Corollary 2.8. If $\left\{T_{i j}\right\}_{\substack{i=1,2, \ldots, k \\ j=1,2, \ldots, n_{i}}} \subset B(\mathcal{H})$ satisfies the relation (2.5), then for any polynomial $p\left(1,\left\{x_{i j}\right\},\left\{y_{i j}\right\}\right) \in \mathcal{P}$,

$$
\left\|p\left(I_{\mathcal{H}},\left\{T_{i j}\right\},\left\{T_{i j}^{*}\right\}\right)\right\| \leq \sup \left\|p\left(I_{\mathcal{K}},\left\{V_{i j}\right\},\left\{V_{i j}^{*}\right\}\right)\right\|,
$$

where the supremum is taken over all sequences of isometries $\left(V_{i 1}, \ldots, V_{i n_{i}}\right)$ $(i=1,2, \ldots, k)$ on a Hilbert space $\mathcal{K}$ such that

$$
V_{i 1} V_{i 1}^{*}+\cdots+V_{i n_{i}} V_{i n_{i}}^{*}=I_{\mathcal{K}}, \quad i=1,2, \ldots, k .
$$

Let us mention here a particular case. A hereditary polynomial in $2 n$ noncommuting indeterminates $\left\{x_{i}\right\},\left\{y_{i}\right\}(i=1,2, \ldots, n)$ has the form

$$
p\left(1,\left\{x_{i}\right\},\left\{y_{i}\right\}\right)=a_{0}+\sum a_{i_{1} \cdots j_{q}} x_{i_{1}} \cdots x_{i_{p}} y_{j_{1}} \cdots y_{j_{q}},
$$

where $a_{0}, a_{i_{1} \cdots j_{q}} \in \mathbf{C}$.

Corollary 2.9. If $\left\{T_{i}\right\}_{i=1}^{n} \subset B(\mathcal{H})$ such that

$$
T_{1} T_{1}^{*}+\cdots T_{n} T_{n}^{*}=I_{\mathcal{H}}
$$

then for any hereditary polynomial $p\left(1,\left\{x_{i}\right\},\left\{y_{i}\right\}\right)$

$$
\left\|p\left(I_{\mathcal{H}},\left\{T_{i}\right\},\left\{T_{i}^{*}\right\}\right)\right\| \leq\left\|p\left(1,\left\{\sigma_{i}\right\},\left\{\sigma_{i}^{*}\right\}\right)\right\|_{\mathcal{O}_{n}},
$$

where $\left\{\sigma_{i}\right\}_{i=1}^{n}$ is a system of generators for the Cuntz algebra $\mathcal{O}_{n}$.

Let us remark that, under the condition (2.8), the inequality (2.9) is sharper than the one obtained in [Po3].

\section{Free product operator algebras and their representations.}

We need a few definitions from $[\mathbf{B}]$. Let $\Gamma$ be a set, and let $n: \Gamma \rightarrow \mathbb{N}$ be a function with $n(\gamma)=n_{\gamma}$. Let $\Lambda$ be a set of variables (or formal symbols) $x_{i j}^{\gamma}$, one variable for each $\gamma \in \Gamma$ and each $i, j, 1 \leq i, j \leq n_{\gamma}$. We call these matrix entry variables, or quantum variables. Let $\mathcal{F}$ be the free associative algebra on $\Lambda$. Let $\mathcal{R}$ be a set of polynomial identities $P=0$ in the variables in $\Lambda$. Regard $\mathcal{R}$ as subset of $\mathcal{F}$. Take a quotient of $\mathcal{F}$ by the ideal generated by $\mathcal{R}$.

We define a semi-norm on $M_{n}(\mathcal{F})$ by

$$
\left\|\left[u_{i j}\right]\right\|_{\Lambda}=\sup \left\{\left\|\left[\pi\left(u_{i j}\right)\right]\right\|\right\}
$$

where the supremum is taken over all algebra representations $\pi$ of $\mathcal{F} / \mathcal{R}$ on a separable Hilbert space satisfying the condition $\left\|\left[\pi\left(x_{i j}^{\gamma}\right)\right]\right\| \leq 1$ for all $\gamma$. This later matrix is indexed on rows by $i$ and on columns by $j$, for all $1 \leq i, j \leq n_{\gamma}$.

Now, quotient by nullspace of this semi-norm to obtain an operator algebra. The completion of this space is denoted by $O A(\Lambda, \mathcal{R})$. This is called the free operator algebra on $\Lambda$ with relations $\mathcal{R}$ (see $[\mathbf{B}]$ ). 
Let $\Delta_{x y}$ have the identity $e$ and also contain the ordinary variables $\left\{x_{i j}\right\}_{\substack{i=1,2, \ldots, k \\ j=1,2, \ldots, n_{i}}}$ and $\left\{y_{i j}\right\}_{\substack{i=1,2, \ldots, k \\ j=1,2, \ldots, n_{i}}}$, and let $\mathcal{R}_{x y}$ be the relations

$$
y_{i \alpha} x_{i \beta}=\delta_{\alpha \beta} e, \text { for any } i=1,2, \ldots, k \text { and } \alpha, \beta=1,2, \ldots, n_{i} .
$$

Form the universal algebra $O A\left(\Delta_{x y}, \mathcal{R}_{x y}\right)$.

Theorem 3.1. The universal algebra $O A\left(\Delta_{x y}, \mathcal{R}_{x y}\right)$ is completely isometrically isomorphic to the amalgamated (over the identity) free product $C^{*}$ algebra ${ }^{*} \mathbf{C} \mathcal{T}_{n_{i}}$.

Proof. According to (3.1), for any polynomials $p_{r s}\left(e,\left\{x_{i j}\right\},\left\{y_{i j}\right\}\right), \quad 1 \leq$ $r, s \leq m$, we have

$$
\left\|\left[p_{r s}\left(e,\left\{x_{i j}\right\},\left\{y_{i j}\right\}\right)\right]\right\|_{\Delta_{x y}}=\sup \left\{\left\|\left[p\left(I_{\mathcal{H}},\left\{A_{i j}\right\},\left\{B_{i j}\right\}\right)\right]\right\|\right\},
$$

where the supremum is taken for all contractions $A_{i j}, B_{i j} \in B(\mathcal{H}) \quad(i=$ $\left.1,2, \ldots, k ; j=1,2, \ldots, n_{i}\right)$ satisfying the relations

$$
B_{i \alpha} A_{i \beta}=\delta_{\alpha \beta} I_{\mathcal{H}}, \quad \text { for any } i=1,2, \ldots, k ; \alpha, \beta=1,2, \ldots, n_{i} .
$$

Under the above conditions, one can prove that $A_{i \beta}^{*}=B_{i \beta}$ (see $[\mathbf{P o 6}]$ ) and consequently $\left(A_{i 1}, \ldots, A_{i n_{i}}\right)$ is a contractive sequence of isometries for each $i=1,2, \ldots, k$. Therefore, the relation (3.2) becomes

$$
\left\|\left[p_{r s}\left(e,\left\{x_{i j}\right\},\left\{y_{i j}\right\}\right)\right]\right\|_{\Delta_{x y}}=\sup \left\{\left\|\left[p\left(I,\left\{V_{i j}\right\},\left\{V_{i j}^{*}\right\}\right)\right]\right\|\right\},
$$

where the supremum is taken for all contractive sequences of isometries $\left(V_{i 1}, \ldots, V_{i n_{i}}\right)(i=1,2, \ldots, k)$ acting on a Hilbert space.

On the other hand, the $*$-representations $\pi$ of $*_{\mathbf{C}} \mathcal{T}_{n_{i}}$ are in one-to-one corespondence with $k$-tuples $\pi_{1}, \ldots, \pi_{k}$ of $*$-representations of $\mathcal{T}_{n_{1}}, \ldots, \mathcal{T}_{n_{k}}$, respectively, on the same Hilbert space, i.e.,

$$
\left.\pi\right|_{\mathcal{T}_{n_{i}}}=\pi_{i}, \quad i=1,2, \ldots, k .
$$

According to $[\mathbf{P o 3}]$, the $*$-representations $\pi_{i}: \mathcal{T}_{n_{i}} \rightarrow B(\mathcal{K})$ are in one-to-one corespondence with the contractive sequences of isometries $\left(V_{i 1}, \ldots, V_{i n_{i}}\right)$ such that $\pi_{i}\left(S_{i j}\right)=V_{i j}$ and $\pi_{i}(1)=I_{\mathcal{K}}$, where $S_{i 1}, \ldots, S_{i n_{i}}$ is a system of generators of the Toeplitz $C^{*}$-algebra $\mathcal{T}_{n_{i}}$. Therefore,

$$
\begin{aligned}
\left\|\left[p_{r s}\left(e,\left\{x_{i j}\right\},\left\{y_{i j}\right\}\right)\right]\right\|_{\Delta_{x y}} & =\sup \left\|\left[p_{r s}\left(I,\left\{\pi_{i}\left(S_{i j}\right)\right\},\left\{\pi_{i}\left(S_{i j}\right)^{*}\right\}\right)\right]\right\| \\
& =\sup \left\|\left[\pi\left(p_{r s}\left(I,\left\{S_{i j}\right\},\left\{S_{i j}^{*}\right\}\right)\right)\right]\right\|,
\end{aligned}
$$

where the supremum is taken over all $*$-representations $\pi$ of $*_{\mathbf{C}} \mathcal{T}_{n_{i}}$ such that (3.4) holds. This shows that $O A\left(\Delta_{x y}, \mathcal{R}_{x y}\right)$ is completely isometrically isomorphic to $\check{*}_{\mathbf{C}} \mathcal{T}_{n_{i}}$, and the proof is complete.

The internal characterization of the matrix norm on a universal algebra $O A(\Lambda, \mathcal{R})$ (see $[\mathbf{B}],[\mathbf{B P}]$ ) leads to the following factorization theorem. 
Theorem 3.2. If $P=\left[p_{r s}\right]_{m \times m}$ is a matrix of polynomials in e, $\left\{x_{i j}\right\},\left\{y_{i j}\right\}$ then, $\|P\|_{\Delta_{x y}}<1$ if and only if there is a positive integer $t$ such that

$$
P=A_{0} D_{1} A_{1} D_{2} \cdots D_{t} A_{t},
$$

where $A_{\ell}(\ell=0,1, \ldots, t)$ are scalar matrices (with a finite number of nonzero entries), each $\left\|A_{\ell}\right\|<1$, and each $D_{\ell}$ is diagonal matrix with $e, x_{i j}, y_{i j}$ $\left(i \in\{1,2, \ldots, k\}\right.$ and $\left.j \in\left\{1,2, \ldots, n_{i}\right\}\right)$ as the diagonal entries.

Blecher and Paulsen defined in $[\mathbf{B P}]$ the free product with amalgamation over $\mathbf{C}$ in the category consisting of unital operator algebras as objects and completely contractive homomorphisms as morphisms. For each $i=$ $1,2, \ldots, k$, let $\mathcal{A}_{n_{i}}$ be the noncommutative disc algebra on $n_{i}$-generators [Po4], and let $\mathcal{A}_{n_{1}} \check{*}_{\mathbf{C}} \cdots \check{*}_{\mathbf{C}} \mathcal{A}_{n_{k}}$ be the amalgamated free product operator algebra. This is the unique unital algebra which has the following universal property: there are unital completely isometric imbeddings

$$
\chi_{i}: \mathcal{A}_{n_{i}} \rightarrow \mathcal{A}_{n_{1} \tilde{*}_{\mathbf{C}}} \cdots \check{*}_{\mathbf{C}} \mathcal{A}_{n_{k}} \quad(i=1,2, \ldots, k)
$$

such that the images of $\mathcal{A}_{n_{i}}(i=1,2, \ldots, k)$ under $\chi_{i}(i=1,2, \ldots, k)$ generate $\mathcal{A}_{n_{1}} \tilde{*}_{\mathbf{C}} \cdots \mathscr{*}_{\mathbf{C}} \mathcal{A}_{n_{k}}$, and if for each $i=1,2, \ldots, k, \pi_{i}$ is a unital completely contractive homomorphism from $\mathcal{A}_{n_{i}}$ into an operator algebra $\mathcal{C}$, then there is a unique unital completely contractive homomorphism

$$
\pi_{1} * \cdots * \pi_{k}: \mathcal{A}_{n_{1}} \check{*}_{\mathbf{C}} \cdots \check{*}_{\mathbf{C}} \mathcal{A}_{n_{k}} \rightarrow \mathcal{C}
$$

with $\left(\pi_{1} * \cdots * \pi_{k}\right) \circ \chi_{i}=\pi_{i} \quad(i=1,2, \ldots, k)$.

Let us denote by $O A_{+}\left(\Delta_{x y}, \mathcal{R}_{x y}\right)$ the closed subalgebra generated by the variables $e,\left\{x_{i j}\right\}_{\substack{i=1,2, \ldots, k \\ j=1,2, \ldots, n_{i}}}$ in $O A\left(\Delta_{x y}, \mathcal{R}_{x y}\right)$.

Theorem 3.3. The amalgamated free product operator algebra $\mathcal{A}_{n_{1}} \tilde{*}_{\mathbf{C}} \cdots$ ${ }^{*} \mathbf{C} \mathcal{A}_{n_{k}}$ is completely isometrically isomorphic to the operator algebra $O A_{+}\left(\Delta_{x y}, \mathcal{R}_{x y}\right)$.

Proof. It is enough to prove that the algebra $O A_{+}\left(\Delta_{x y}, \mathcal{R}_{x y}\right)$ has the abovementioned universal property. Let $\left\{S_{i 1}, \ldots, S_{i n_{i}}\right\}$ be a system of generators of $\mathcal{A}_{n_{i}}(i=1,2, \ldots, k)$. According to the von Neumann inequality (see $[\mathbf{P o 2}],[\mathbf{P o} 4])$, one can easily see that the homomorphism

$$
\chi_{i}: \mathcal{A}_{n_{i}} \rightarrow O A_{+}\left(\Delta_{x y}, \mathcal{R}_{x y}\right)
$$

defined by $\chi_{i}\left(S_{i j}\right)=x_{i j} \quad\left(j=1,2, \ldots, n_{i}\right)$ is a unital completely isometric imbedding, and $\chi_{i}\left(\mathcal{A}_{n_{i}}\right) \quad(i=1,2, \ldots, k)$ generate $O A_{+}\left(\Delta_{x y}, \mathcal{R}_{x y}\right)$. Moreover, if $\mathcal{C}$ is an operator algebra and

$$
\pi_{i}: \mathcal{A}_{n_{i}} \rightarrow \mathcal{C} \quad(i=1,2, \ldots, k)
$$


are unital completely contractive homomorphism, then according to [Po4], there exists $\left\{T_{i j}\right\}_{\substack{i=1,2, \ldots, k \\ j=1,2, \ldots, n_{i}}} \subset \mathcal{C}$ such that $\left(T_{i 1}, \ldots, T_{i n_{i}}\right)$ is a contractive sequence of operators for each $i=1,2, \ldots, k$, and $\pi_{i}\left(S_{i j}\right)=T_{i j}$. Define

$$
\left(\pi_{1} * \cdots * \pi_{k}\right)\left(p\left(e,\left\{x_{i j}\right\}\right)\right)=p\left(I,\left\{T_{i j}\right\}\right)
$$

for any polynomial in $e,\left\{x_{i j}\right\}_{\substack{i=1,2, \ldots, k \\ j=1,2, \ldots, n_{i}}}$. Taking into account the definition of $O A_{+}\left(\Delta_{x y}, \mathcal{R}_{x y}\right)$, Theorem 3.1, and the von Neumann inequality (2.3), we infer that $\pi_{1} * \cdots * \pi_{k}$ is contractive and can be uniquely extended to a unital contractive homomorphism

$$
\pi_{1} * \cdots * \pi_{k}: O A_{+}\left(\Delta_{x y}, \mathcal{R}_{x y}\right) \rightarrow \mathcal{C}
$$

such that $\left(\pi_{1} * \cdots * \pi_{k}\right)\left(\chi_{i}\left(S_{i j}\right)\right)=T_{i j}$. The proof is complete.

Using Theorem 3.1 one can deduce the following.

Corollary 3.4. The operator algebra $\mathcal{A}_{n_{1}} \check{*}_{\mathbf{C}} \cdots \check{*}_{\mathbf{C}} \mathcal{A}_{n_{k}}$ is completely isometrically embedded in the $C^{*}$-algebra $\check{*}_{\mathbf{C}} \mathcal{T}_{n_{i}}$.

Remark 3.5. The result from Theorem 3.3 can be also obtained using the results from $[\mathbf{B o}],[\mathbf{B P}]$, and $[\mathbf{P o} 4]$.

Let $\mathcal{P}_{x}$ be the set of all polynomials in the noncommuting indeterminates $\left\{x_{i j}\right\}_{\substack{i=1,2, \ldots, k \\ j=1,2, \ldots, n_{i}}}$. Notice that $\mathcal{P}_{x} \subset \mathcal{P}(\mathcal{P}$ was introduced in Section 1$)$, and any $p \in \mathcal{P}$ can be viewed as an element in $O A\left(\Delta_{x y}, \mathcal{R}_{x y}\right)$.

Theorem 3.6. Let $\left\{A_{i j}\right\}_{\substack{i=1,2, \ldots, k \\ j=1,2, \ldots, n_{i}}}$ be in $B(\mathcal{H})$. Then $\left(A_{i 1}, \ldots, A_{i n_{i}}\right)$ is a contractive sequence of operators for each $i=1,2, \ldots, k$, if and only if the map

$$
\Phi: \mathcal{P}_{x} \subset O A_{+}\left(\Delta_{x y}, \mathcal{R}_{x y}\right) \rightarrow B(\mathcal{H})
$$

defined by $\Phi\left(p\left(e,\left\{x_{i j}\right\}\right)\right)=p\left(I_{\mathcal{H}},\left\{A_{i j}\right\}\right)$ is a completely contractive homomorphism.

Proof. Assume that $\left(A_{i 1}, \ldots, A_{i n_{i}}\right)$ is contractive for each $i=1,2, \ldots, k$. According to Theorem 2.1, there is a Hilbert space $\mathcal{K} \supset \mathcal{H}$ and contractive sequences $\left(V_{i 1}, \ldots, V_{i n_{i}}\right)$ of isometries on $\mathcal{K}$ such that

$$
\left.V_{i j}^{*}\right|_{\mathcal{H}}=A_{i j}^{*}, \quad i=1,2, \ldots, k ; j=1,2, \ldots, n_{i} .
$$

The map $\Psi: O A\left(\Delta_{x y}, \mathcal{R}_{x y}\right) \rightarrow C^{*}\left(\left\{V_{i j}\right\}\right)$ defined by

$$
\Psi\left(p\left(e,\left\{x_{i j}\right\},\left\{y_{i j}\right\}\right)\right)=p\left(I_{\mathcal{K}},\left\{V_{i j}\right\},\left\{V_{i j}^{*}\right\}\right),
$$

where $p\left(e,\left\{x_{i j}\right\},\left\{y_{i j}\right\}\right) \in \mathcal{P}$, is completely contractive (see Theorem 3.1). Therefore, $\|\Psi\|_{c b} \leq 1$. According to (3.6), we have

$$
\begin{aligned}
\Phi\left(p\left(e,\left\{x_{i j}\right\}\right)\right) & =p\left(I_{\mathcal{K}},\left\{A_{i j}\right\}\right)=\left.P_{\mathcal{H}} p\left(I_{\mathcal{K}},\left\{V_{i j}\right\}\right)\right|_{\mathcal{H}} \\
& =\left.P_{\mathcal{H}} \Psi\left(p\left(e,\left\{x_{i j}\right\}\right)\right)\right|_{\mathcal{H}},
\end{aligned}
$$


for any $p\left(e,\left\{x_{i j}\right\}\right) \in O A_{+}\left(\Delta_{x y}, \mathcal{R}_{x y}\right)$. Therefore, $\|\Phi\|_{c b} \leq\|\Psi\|_{c b} \leq 1$.

Conversely, suppose $\left\{A_{i j}\right\}_{\substack{i=1,2, \ldots, k \\ j=1,2, \ldots, n_{i}}} \subset B(\mathcal{H})$ such that map

$$
\Phi: \mathcal{P}_{x} \subset O A_{+}\left(\Delta_{x y}, \mathcal{R}_{x y}\right) \rightarrow B(\mathcal{H})
$$

defined by $\Phi\left(p\left(e,\left\{x_{i j}\right\}\right)\right)=p\left(I_{\mathcal{H}},\left\{A_{i j}\right\}\right)$ is completely contractive. In particular, for each $i=1,2, \ldots, k$, we have

$$
\left\|\left[\begin{array}{cccc}
A_{i 1} & A_{i 2} & \cdots & A_{i n_{i}} \\
0 & 0 & \cdots & 0 \\
\vdots & \vdots & & \vdots \\
0 & 0 & \cdots & 0
\end{array}\right]\right\| \leq\left\|\left[\begin{array}{cccc}
x_{i 1} & x_{i 2} & \cdots & x_{i n_{i}} \\
0 & 0 & \cdots & 0 \\
\vdots & \vdots & & \vdots \\
0 & 0 & \cdots & 0
\end{array}\right]\right\|_{\Delta_{x}} \leq 1 .
$$

Hence, $\left\|\sum_{i=1}^{n_{i}} A_{i j} A_{i j}^{*}\right\| \leq 1$ for each $i=1,2, \ldots, k$. This completes the proof.

The above theorem and Theorem 3.3 show also that the universal algebra generated by a finite number of contractive sequences of operators on a Hilbert space and the identity is completely isometrically isomorphic to the amalgamated free product operator algebra $\mathcal{A}_{n_{1}{ }^{*} \mathbf{C}} \cdots \check{*}_{\mathbf{*}} \mathcal{A}_{n_{k}}$ for some integers $n_{1}, \ldots, n_{k} \geq 1$, in the following sense. Given any contractive sequences $\left(T_{i 1}, \ldots, T_{i n_{i}}\right) \quad(i=1,2, \ldots, k)$ of operators on a Hilbert space $\mathcal{H}$, there is a completely contractive homomorphism

$$
\Phi: \mathcal{A}_{n_{1}} \check{*}_{\mathbf{C}} \cdots \check{*}_{\mathbf{C}} \mathcal{A}_{n_{k}} \rightarrow \mathcal{B}(\mathcal{H})
$$

such that $\Phi(1)=1$ and $\Phi\left(x_{i j}\right)=T_{i j}$ for any $i=1,2, \ldots, k$ and $j=$ $1,2, \ldots, n_{i}$. Moreover, this property characterizes $\mathcal{A}_{n} \check{*}_{\mathbf{C}} \cdots \check{*}_{\mathbf{C}} \mathcal{A}_{n_{k}}$ up to unital complete isometric isomorphism.

Similarly to the proof of Theorem 3.6, one can prove the following result.

Theorem 3.7. Let $\left\{A_{i j}\right\}_{\substack{i=1,2, \ldots, k \\ j=1,2, \ldots, n_{i}}} \subset B(\mathcal{H})$. Then $\left(A_{i 1}, \ldots, A_{i n_{i}}\right)$ is a contractive sequence of operators for each $i=1,2, \ldots, k$, if and only if the map

$$
\Psi: \mathcal{P} \subset O A\left(\Delta_{x y}, \mathcal{R}_{x y}\right) \rightarrow B(\mathcal{H})
$$

defined by

$$
\Psi\left(p\left(\left\{x_{i j}\right\},\left\{y_{i j}\right\}\right)\right)=p\left(\left\{A_{i j}\right\},\left\{A_{i j}^{*}\right\}\right)
$$

is completely positive.

Now, using Theorem 3.1 and Theorem 3.7, we infer the following extension of the von Neumann inequality [vN], [Po2], [Po3].

Corollary 3.8. If $\left\{T_{i j}\right\}_{\substack{i=1,2, \ldots, k \\ j=1,2, \ldots, n_{i}}} \subset B(\mathcal{H})$ such that

$$
T_{i 1} T_{i 1}^{*}+\cdots+T_{i n_{i}} T_{i n_{i}}^{*} \leq I_{\mathcal{H}}, \quad i=1,2, \ldots, k,
$$


then for any $p \in \mathcal{P} \subset O A\left(\Delta_{x y}, \mathcal{R}_{x y}\right)$,

$$
\left\|p\left(I_{\mathcal{H}},\left\{T_{i j}\right\},\left\{T_{i j}^{*}\right\}\right)\right\| \leq\|p\|_{\check{*}_{\mathbf{C}}} \mathcal{T}_{n_{i}},
$$

where $p$ is viewed as an element of free product $C^{*}$-algebra ${ }_{*} \mathbf{C} \mathcal{T}_{n_{i}}$.

Using Theorem 3.6 and a well-known result of Paulsen ([P1], [Po2]), one can easily infer the following.

Theorem 3.9. Let $\left\{A_{i j}\right\}_{\substack{i=1,2, \ldots, k \\ j=1,2, \ldots, n_{i}}} \subset B(\mathcal{H})$. The following statements are equivalent.

(i) The map $\Phi: O A_{+}\left(\Delta_{x y}, \mathcal{R}_{x y}\right) \rightarrow B(\mathcal{H})$ defined by

$$
\Phi\left(p\left(e,\left\{x_{i j}\right\}\right)\right)=p\left(I,\left\{A_{i j}\right\}\right)
$$

is completely bounded.

(ii) There is a sequence $\left\{T_{i j}\right\}_{\substack{i=1,2, \ldots, k \\ j=1,2, \ldots, n_{i}}} \subset B(\mathcal{H})$ such that $\left(T_{i 1}, \ldots, T_{i n_{i}}\right)$ $(i=1,2, \ldots, k)$ is contractive, and an invertible operator $S$ satisfying

$$
A_{i j}=S^{-1} T_{i j} S, \quad \text { for any } i=1,2, \ldots, k ; j=1,2, \ldots, n_{i} .
$$

Let $\Delta_{x^{\prime} y^{\prime}}$ have the identity $e$ and also contain the ordinary variables $\left\{x_{i j}^{\prime}\right\}_{\substack{i=1,2, \ldots, k \\ j=1,2, \ldots, n_{i}}}$ and $\left\{y_{i j}^{\prime}\right\}_{\substack{i=1,2, \ldots, k \\ j=1,2, \ldots, n_{i}}}$, and let $\mathcal{R}_{x^{\prime} y^{\prime}}$ be the relations

$$
y_{i \alpha}^{\prime} x_{i \beta}^{\prime}=\delta_{\alpha \beta} e \text { for any } i=1,2, \ldots, k \text { and } \alpha, \beta=1,2, \ldots, n_{i},
$$

and

$$
x_{i 1}^{\prime} y_{i 1}^{\prime}+\cdots+x_{i n_{i}}^{\prime} y_{i n_{i}}^{\prime}=e, \quad i=1,2, \ldots, k .
$$

Form the universal algebra $O A\left(\Delta_{x^{\prime} y^{\prime}}, \mathcal{R}_{x^{\prime} y^{\prime}}\right)$. One can prove that

$$
O A\left(\Delta_{x^{\prime} y^{\prime}}, \mathcal{R}_{x^{\prime} y^{\prime}}\right)=\check{*}_{\mathbf{C}} \mathcal{O}_{n_{i}} .
$$

The proof is similar to that of Theorem 3.1, so we will omit it. Let us denote by $O A_{+}\left(\Delta_{x^{\prime} y^{\prime}}, \mathcal{R}_{x^{\prime} y^{\prime}}\right)$ the closed subalgebra generated by $e,\left\{x_{i j}^{\prime}\right\}_{\substack{i=1,2, \ldots, k \\ j=1,2, \ldots, n_{i}}}$ in $O A\left(\Delta_{x^{\prime} y^{\prime}}, \mathcal{R}_{x^{\prime} y^{\prime}}\right)$. Using Theorem 2.5 , we can deduce the following.

Corollary 3.10. If $\left\{T_{i j}\right\}_{\substack{i=1,2, \ldots, k \\ j=1,2, \ldots, n_{i}}} \subset B(\mathcal{H})$ such that $\left(T_{i 1}, \ldots, T_{i n_{i}}\right)$ is contractive for each $i=1,2, \ldots, 1$, then the map

$$
\Phi: O A_{+}\left(\Delta_{x^{\prime} y^{\prime}}, \mathcal{R}_{x^{\prime} y^{\prime}}\right) \rightarrow \mathcal{B}(\mathcal{H})
$$

defined by $\Phi\left(p\left(e,\left\{x_{i j}^{\prime}\right\}\right)\right)=p\left(I_{\mathcal{H}},\left\{T_{i j}\right\}\right)$ is a completely contractive homomorphism.

Due to the universal property of $\mathcal{A}_{n_{1}} \check{*}_{\mathbf{C}} \cdots \check{*}_{\mathbf{C}} \mathcal{A}_{n_{k}}$, one can deduce the following.

Theorem 3.11. The amalgamated free product operator algebra $\mathcal{A}_{n_{1}} \tilde{*}_{\mathbf{C}} \cdots$ ${ }^{*} \mathbf{C} \mathcal{A}_{n_{k}}$ is completely isometrically isomorphic to $O A_{+}\left(\Delta_{x^{\prime} y^{\prime}}, \mathcal{R}_{x^{\prime} y^{\prime}}\right)$. 
Now, the inequality (1.4) announced in Section 1, follows from Corollary 3.8 and Theorem 3.11.

Corollary 3.12. If $\left\{T_{i j}\right\}_{\substack{i=1,2, \ldots, k \\ j=1,2, \ldots, n_{i}}} \in B(\mathcal{H})$ such that

$$
T_{i 1} T_{i 1}^{*}+\cdots+T_{i n_{i}} T_{i n_{i}}^{*} \leq I_{\mathcal{H}}, \quad i=1,2, \ldots, k,
$$

then for any polynomial $q\left(1,\left\{x_{i j}\right\}\right) \in \mathcal{P}$,

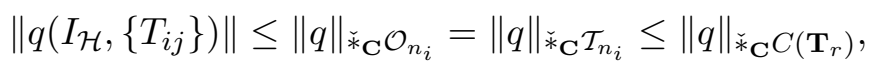

where $\mathbf{T}_{r}=\mathbf{T}\left(r=1,2, \ldots, n_{1}+\cdots n_{k}\right)$, and $q\left(1,\left\{x_{i j}\right\}\right)$ is seen as an element of $\check{*}_{\mathbf{C}} \mathcal{O}_{n_{i}}, \check{*}_{\mathbf{C}} \mathcal{T}_{n_{i}}$, and $\check{*}_{\mathbf{C}} C\left(\mathbf{T}_{r}\right)$, respectively.

The inequality

$$
\left\|q\left(I_{\mathcal{H}},\left\{T_{i j}\right\}\right)\right\| \leq\|q\|_{\text {F }_{\mathbf{C}} C\left(\mathbf{T}_{r}\right)}
$$

was proved by Bozejko in $[\mathbf{B o z}]$ (see also [Bo]) and follows also from Corollary 2.6. Notice that, in our setting, the inequality (3.7) is sharper than (3.8).

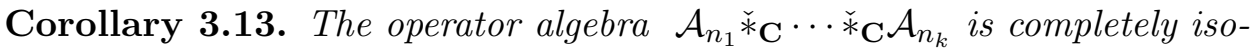
metrically imbedded in the $C^{*}$-algebra $\stackrel{*}{\mathrm{C}}_{\mathrm{O}} \mathrm{O}_{n_{i}}$.

Using Theorem 2.7 and Corollary 3.8 one can infer the following version of the von Neumann inequality.

Corollary 3.14. If $\left\{T_{i j}\right\}_{\substack{i=1,2, \ldots, k \\ j=1,2, \ldots, n_{i}}} \in B(\mathcal{H})$ such that

$$
T_{i 1} T_{i 1}^{*}+\cdots+T_{i n_{i}} T_{i n_{i}}^{*}=I_{\mathcal{H}}, \quad i=1,2, \ldots, k,
$$

then for any polynomial $q\left(1,\left\{x_{i j}\right\},\left\{y_{i j}\right\}\right) \in \mathcal{P}$,

$$
\left\|q\left(I_{\mathcal{H}},\left\{T_{i j}\right\},\left\{T_{i j}^{*}\right\}\right)\right\| \leq\|q\|_{\check{*}_{\mathbf{C}} \mathcal{O}_{n_{i}}} \leq\|q\|_{\check{*}_{\mathbf{C}} \mathcal{T}_{n_{i}}},
$$

where $q\left(1,\left\{x_{i j},\left\{y_{i j}\right\}\right\}\right)$ is seen as an element of $\check{*}_{\mathbf{C}} \mathcal{O}_{n_{i}}$ and $\check{*}_{\mathbf{C}} \mathcal{T}_{n_{i}}$, respectively.

Let $\Delta_{x}$ have the entries in the row matrices $\left[x_{i 1}, \ldots, x_{i n_{i}}\right] \quad(i=1,2, \ldots, k)$ (so there are some relations forcing the other entries to be zero) and also an identity $e$ (i.e., $x_{i j} e=e x_{i j}$ for $i=1,2, \ldots, k$ and $j=1,2, \ldots, n_{i}$ ). Consider the universal algebra $O A\left(\Delta_{x}, \mathcal{R}_{x}\right)$.

Theorem 3.15. The universal algebra $O A\left(\Delta_{x}, \mathcal{R}_{x}\right)$ is completely isometrically isomorphic to $O A_{+}\left(\Delta_{x y}, \mathcal{R}_{x y}\right)$.

Proof. According to (3.1), for any polynomials $p_{r s}\left(e,\left\{x_{i j}\right\}\right), 1 \leq r, s \leq m$, we have

$$
\left\|\left[p_{r s}\left(e,\left\{x_{i j}\right\}\right)\right]\right\|_{\Delta_{x}}=\sup \left\{\left\|\left[p_{r s}\left(I_{\mathcal{H}},\left\{T_{i j}\right\}\right)\right]\right\|\right\}
$$


where the sumpremum is taken for all $\left\{T_{i j}\right\}_{\substack{i=1,2, \ldots, k \\ j=1,2, \ldots, n_{i}}} \subset \mathcal{B}(\mathcal{H})$ such that $\left(T_{i 1}, \ldots, T_{i n_{i}}\right)(i=1,2, \ldots, k)$ is contractive. According to Theorem 2.1, we infer that

$$
\left\|\left[p_{r s}\left(e,\left\{x_{i j}\right\}\right)\right]\right\|_{\Delta_{x}}=\sup \left\{\left\|\left[p_{r s}\left(I,\left\{V_{i j}\right\}\right)\right]\right\|\right\},
$$

where the supremum is taken for all contractive sequences of isometries $\left(V_{i 1}, \ldots, V_{i n_{i}}\right)(i=1,2, \ldots, k)$ acting on a Hilbert space. Using the relation (3.3) we deduce that

$$
\left\|\left[p_{r s}\left(e,\left\{x_{i j}\right\}\right)\right]\right\|_{\Delta_{x}}=\left\|\left[p_{r s}\left(e,\left\{x_{i j}\right\}\right)\right]\right\|_{\Delta_{x y}},
$$

which completes the proof.

Since $\check{*} \mathbf{C} \mathcal{A}_{n_{i}}$ and $\check{*}_{\mathbf{C}} \mathcal{O}_{n_{i}}$ are universal algebras of type $O A(\Lambda, \mathcal{R})$, one can obtain factorizations of type (3.5) in a similar manner.

On the other hand, let us remark that all the von Neumann inequalities presented in this section can be easily extended to matrices.

\section{Characters on free product disc algebras and cohomology.}

Let $\lambda=\left\{\lambda_{i j}\right\}_{\substack{i=1,2, \ldots, k \\ j=1,2, \ldots, n_{i}}}$ be a sequence of complex numbers such that

$$
\left|\lambda_{i 1}\right|^{2}+\cdots+\left|\lambda_{i n_{i}}\right|^{2} \leq 1 \quad \text { for each } i=1,2, \ldots, k,
$$

and define the "evaluation" functional

$$
\Phi_{\lambda}: \mathcal{P}_{x} \rightarrow \mathbf{C} ; \quad \Phi_{\lambda}\left(p\left(e,\left\{x_{i j}\right\}\right)\right)=p\left(1,\left\{\lambda_{i j}\right\}\right),
$$

where $\mathcal{P}_{x}$ is the set of all polynomials $p\left(e,\left\{x_{i j}\right\}\right) \in O A_{+}\left(\Delta_{x y}, \mathcal{R}_{x y}\right)$. According to Theorem 3.7, we have

$$
\left|p\left(1,\left\{\lambda_{i j}\right\}\right)\right| \leq\left\|p\left(e,\left\{x_{i j}\right\}\right)\right\|_{O A_{+}\left(\Delta_{x y}, \mathcal{R}_{x y}\right)} .
$$

Hence, $\Phi_{\lambda}$ has a unique extension to $O A_{+}\left(\Delta_{x y}, \mathcal{R}_{x y}\right)$ Therefore $\Phi_{\lambda}$ is a character on $O A_{+}\left(\Delta_{x y}, \mathcal{R}_{x y}\right)$. Let $M_{O A_{+}\left(\Delta_{x y}, \mathcal{R}_{x y}\right)}$ be the set of all characters of $O A_{+}\left(\Delta_{x y}, \mathcal{R}_{x y}\right)$ and let

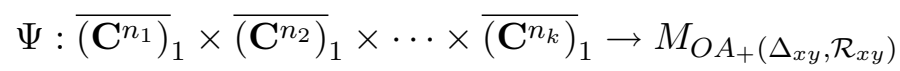

be defined by $\Psi(\lambda)=\Phi_{\lambda}$, where $\lambda=\left\{\lambda_{i j}\right\}_{\substack{i=1,2, \ldots, k \\ j=1,2, \ldots, n_{i}}}$.

Theorem 4.1. The map $\Psi$ is a homeomorphism of $\overline{\left(\mathbf{C}^{n_{1}}\right)_{1}} \times \cdots \times \overline{\left(\mathbf{C}^{n_{k}}\right)}{ }_{1}$ onto $M_{O A_{+}\left(\Delta_{x y}, \mathcal{R}_{x y}\right)}$.

Proof. Let us show that $\Psi$ is one-to-one. If $\lambda=\left\{\lambda_{i j}\right\}_{\substack{i=1,2, \ldots, k \\ j=1,2, \ldots, n_{i}}}$ and $\mu=$

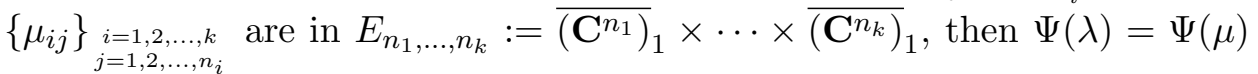
implies

$$
\lambda_{i j}=\Phi_{\lambda}\left(x_{i j}\right)=\Phi_{\mu}\left(x_{i j}\right)=\mu_{i j}
$$


for any $i=1,2, \ldots k, j=1,2, \ldots, n_{i}$. Therefore $\lambda=\mu$. Now, assume that $\Phi: O A_{+}\left(\Delta_{x y}, \mathcal{R}_{x y}\right) \rightarrow \mathbf{C}$ is a character. Setting $\Phi\left(x_{i j}\right)=\lambda_{i j} \in \mathbf{C}$ we have

$$
\Phi\left(p\left(\left\{x_{i j}\right\}\right)\right)=p\left(\left\{\lambda_{i j}\right\}\right),
$$

for any $p\left(\left\{x_{i j}\right\}\right) \in O A_{+}\left(\Delta_{x y}, \mathcal{R}_{x y}\right)$. Since $\Phi$ is a character it follows that it is completely contractive. Applying Theorem 3.7 when $A_{i j}=\lambda_{i j} I_{\mathbf{C}}, i=$ $1,2, \ldots, k, j=1,2, \ldots, n_{i}$, we infer that $\left\{\lambda_{i j}\right\} \in E_{n_{1}, \ldots, n_{k}}$.

On the other hand, the identity

$$
\Phi\left(p\left(\left\{x_{i j}\right\}\right)\right)=p\left(\lambda_{i j}\right)=\Phi_{\lambda}\left(p\left(\left\{x_{i j}\right\}\right)\right)
$$

proves that $\Phi=\Phi_{\lambda}$ on the subset $\mathcal{P}_{x}$ which is dense in $O A_{+}\left(\Delta_{x y}, \mathcal{R}_{x y}\right)$. Hence $\Phi=\Phi_{\lambda}$. Since both $E_{n_{1}, \ldots, n_{k}}$ and $M_{O A_{+}\left(\Delta_{x y}, \mathcal{R}_{x y}\right)}$ are compact Hausdorff spaces and $\Psi$ is one-to-one and onto, to complete the proof it suffices to show that $\Phi$ is continuous.

Suppose that $\lambda^{\alpha}=\left\{\lambda_{i j}^{\alpha}\right\}(\alpha \in J)$ is a net in $E_{n_{1}, \ldots, n_{k}}$ such that $\lim _{\alpha \in J} \lambda^{\alpha}=$ $\lambda=\left\{\lambda_{i j}\right\}$. Since $\sup _{\alpha \in J}\left\|\Phi_{\lambda^{\alpha}}\right\| \leq 1$ and $\mathcal{P}_{x}$ is dense in $O A_{+}\left(\Delta_{x y}, \mathcal{R}_{x y}\right)$ and since

$$
\lim _{\alpha \in J} \Phi_{\lambda^{\alpha}}\left(p\left(\left\{x_{i j}\right\}\right)\right)=\lim _{\alpha \in J} p\left(\left\{\lambda_{i j}\right\}\right)=\Phi_{\lambda}\left(p\left(\left\{x_{i j}\right\}\right)\right)
$$

for every $p\left(\left\{x_{i j}\right\}\right) \in \mathcal{P}_{x}$, it follows that $\Psi$ is continuous. The proof is complete.

Let us remark that in the particular case when $k=1, n_{1}=n$ we get $M_{\mathcal{A}_{n}}=\left(\overline{\mathbf{C}^{n}}\right)_{1}\left(\mathcal{A}_{n}\right.$ is the noncommutative disc algebra [Po2] $)$, result that was obtained in [Po4].

Let $A$ be a complex Banach algebra with unit, $X$ be a Banach $A$-bimodule, and $X^{\prime}$ be the dual Banach $A$-bimodule (see $[\mathrm{BD}]$ ). We need to recall from $[\mathrm{BD}]$ a few definitions.

A bounded $X$-derivation is a bounded linear mapping $D$ of $A$ into $X$ such that

$$
D(a b)=(D a) b+a(D b), \quad \text { for any } a, b \in A .
$$

The set of all bounded $X$-derivations is denoted by $Z^{1}(A, X)$. For each $x \in X$ let us define $\delta_{x}: A \rightarrow X$ by $\delta_{x}(a)=a x-x a$. We call $\delta_{x}$ an inner $X$ derivation, and denote by $B^{1}(A, X)$ the set of all inner $X$-derivations. The quotient space $Z^{1}(A, X) / B^{1}(A, X)$ is called the first cohomology group of $A$ with coefficients in $X$, and it is denoted by $H^{1}(A, X)$. A Banach algebra $A$ is said to be amenable if $H^{1}\left(A, X^{\prime}\right)=\{0\}$ for every Banach $A$-bimodule $X$.

It is clear that $\mathbf{C}$, the set of all complex numbers, is a Banach $O A_{+}\left(\Delta_{x y}, \mathcal{R}_{x y}\right)$-bimodule under the module multiplication

$$
\lambda \cdot f\left(\left\{x_{i j}\right\}\right)=f\left(\left\{x_{i j}\right\}\right) \cdot \lambda=\lambda f(\{0\})
$$


for each $f\left(\left\{x_{i j}\right\}\right) \in O A_{+}\left(\Delta_{x y}, \mathcal{R}_{x y}\right)$. According to the von Neumann inequality (3.5), we infer that $\left|\lambda \cdot f\left(\left\{x_{i j}\right\}\right)\right| \leq|\lambda|\left\|f\left(\left\{x_{i j}\right\}\right)\right\|$, for any $\lambda \in \mathbf{C}$ and $f\left(\left\{x_{i j}\right\}\right) \in O A_{+}\left(\Delta_{x y}, \mathcal{R}_{x y}\right)$.

Since the proof of the following theorem is a straightforward extension of [Po4, Theorem 4.1], we will omit it.

Theorem 4.2. The first cohomology group of $O A_{+}\left(\Delta_{x y}, \mathcal{R}_{x y}\right)$ with complex coefficients is isomorphic to the additive group $\mathbf{C}^{n_{1}+n_{2}+\cdots+n_{k}}$.

Since $\mathbf{C}$ is a dual bimodule we infer the following.

Corollary 4.3. The free product operator algebra $O A_{+}\left(\Delta_{x y}, \mathcal{R}_{x y}\right)$ is not amenable.

\section{Positive definite operator-valued kernels on free semigroups.}

Let $\mathbf{F}_{n}^{+}$be the unital semigroup on $n$ generators. A positive definite kernel on $\mathbf{F}_{n}^{+}$is a map

$$
K: \mathbf{F}_{n}^{+} \times \mathbf{F}_{n}^{+} \rightarrow B(\mathcal{H})
$$

with the property that $K(\sigma, \omega)=K(\omega, \sigma)^{*},\left(\sigma, \omega \in \mathbf{F}_{n}^{+}\right)$and

$$
\sum_{i, j=1}^{k}\left\langle K\left(\sigma_{i}, \sigma_{j}\right) h_{j}, h_{i}\right\rangle \geq 0
$$

for any $k \in \mathbf{N}$, for any $h_{1}, \ldots, h_{k} \in \mathcal{H}$, and $\sigma_{1}, \ldots, \sigma_{k} \in \mathbf{F}_{n}^{+}$. A kernel $K$ on $\mathbf{F}_{n}^{+}$is called Toeplitz if $K(e, e)=I_{\mathcal{H}}$ and

$$
K(\alpha \sigma, \alpha \omega)=K(\sigma, \omega) \text { for any } \alpha, \sigma, \omega \in \mathbf{F}_{n}^{+}
$$

(see [Po5] for a particular case). We say that $K$ has a Naimark dilation if there is a Hilbert space $\mathcal{K} \supset \mathcal{H}$ and $\left\{V_{\sigma}\right\}_{\sigma \in \mathbf{F}_{n}^{+}}$a semigroup of isometries on $\mathcal{K}$, i.e., $V_{\sigma} V_{\omega}=V_{\sigma \omega}\left(\sigma, \omega \in \mathbf{F}_{n}^{+}\right), V_{e}=I_{\mathcal{K}}$, such that

$$
K(\sigma, \omega)=\left.P_{\mathcal{H}} V_{\sigma}^{*} V_{\omega}\right|_{\mathcal{H}} \quad \text { for any } \sigma, \omega \in \mathbf{F}_{n}^{+},
$$

where $P_{\mathcal{H}}$ is the orthogonal projection of $\mathcal{K}$ onto $\mathcal{H}$. The Naimark dilation is called minimal if $\mathcal{K}=\bigvee_{\sigma \in \mathbf{F}_{n}^{+}} V_{\sigma} \mathcal{H}$.

The following result is an extension of the Naimark dilation $[\mathbf{N}],[\mathbf{S z F} 2]$ to free semigroups. The proof is similar to that of Theorem 2.1 from [Po5], so we will omit it. However, let us point out that in $[\mathbf{P o 5}]$ we considered just a particular Toeplitz kernel. Here, we have a more general setting.

Theorem 5.1. A Toeplitz kernel on $\mathbf{F}_{n}^{+}$is positive definite if and only if it admits a minimal Naimark dilation. Moreover, the minimal Naimark dilation is unique up to an isomorphism.

Let $\mathbf{F}_{n_{i}}^{+}(i=1,2, \ldots, k)$ be the unital free semigroup on $n_{i}$ generators: $s_{i 1}, s_{i 2}, \ldots, s_{i n_{i}}$, and let $e$ be the neutral element. Then $\mathbf{F}_{n}^{+}:=\mathbf{F}_{n_{1}}^{+} * \cdots * \mathbf{F}_{n_{k}}^{+}$is 
the unital free semigroup on $n=n_{1}+\cdots+n_{k}$ generators. If $\left\{T_{i j}\right\}_{\substack{i=1,2, \ldots, k \\ j=1,2, \ldots, n_{i}}} \subset$ $B(\mathcal{H})$, then for each $\sigma=s_{i_{1} j_{1}} \cdots s_{i_{p} j_{p}} \in \mathbf{F}_{n}^{+}$denote $T_{\sigma}:=T_{i_{1} j_{1}} \cdots T_{i_{p} j_{p}}$, and $T_{\sigma}:=I_{\mathcal{H}}$ if $\sigma=e$.

For any $\sigma, \omega \in \mathbf{F}_{n}^{+}$, let us denote by $\operatorname{gld}(\sigma, \omega)$ the greatest left common divisor of them. Therefore,

$$
\sigma=\operatorname{gld}(\sigma, \omega) \alpha \text { and } \omega=\operatorname{gld}(\sigma, \omega) \beta \text { for some } \alpha, \beta \in \mathbf{F}_{n}^{+},
$$

and $\operatorname{gld}(\alpha, \beta)=e$. Notice that to each pair $(\sigma, \omega) \in \mathbf{F}_{n}^{+} \times \mathbf{F}_{n}^{+}$corresponds a unique pair $(\alpha, \beta) \in \mathbf{F}_{n}^{+} \times \mathbf{F}_{n}^{+}$with the above mentioned properties.

Let us define the kernel $K_{c}: \mathbf{F}_{n}^{+} \times \mathbf{F}_{n}^{+} \rightarrow B(\mathcal{H})$ by $K_{c}(\sigma, \omega)=0$ if $\alpha \neq e, \beta \neq e$, and both words $\alpha, \beta$ start with some generators of the same semigroup $\mathbf{F}_{n_{i}}^{+}$, for some $i=1,2, \ldots, k$, and $K_{c}(\sigma, \omega)=T_{\alpha}^{*} T_{\beta}$ otherwise. It is clear that $K_{c}$ is a Toeplitz kernel. Notice also that if $j_{1} \neq j_{2}$, then

$$
K_{c}\left(s_{i j_{1}} \sigma, s_{i j_{2}} \omega\right)=0
$$

for any $\sigma, \omega \in \mathbf{F}_{n}^{+}$.

Theorem 5.2. Let $\left\{T_{i j}\right\}_{\substack{i=1,2, \ldots, k \\ j=1,2, \ldots, n_{i}}} \subset B(\mathcal{H})$. Then $\left(T_{i 1}, \ldots, T_{i n_{i}}\right)$ is a contractive sequence of operators for each $i=1,2, \ldots, k$ if and only if the Toeplitz kernel $\mathcal{K}_{c}$ is positive definite.

Proof. Suppose that for each $i=1,2, \ldots, k$ the sequence $\left(T_{i 1}, \ldots, T_{i n_{i}}\right)$ is contractive. According to Theorem 2.1, there exists a Hilbert space $\mathcal{K} \supset \mathcal{H}$ and contractive sequences $\left(V_{i 1}, \ldots, V_{i n_{i}}\right)(i=1,2, \ldots, k)$ of isometries on $\mathcal{K}$ such that $\left.V_{i j}^{*}\right|_{\mathcal{H}}=T_{i j}^{*}\left(i=1, \ldots, k ; j=1, \ldots, n_{i}\right)$ and $P_{\mathcal{K} \ominus \mathcal{H}} V_{i_{1} j_{1}} \mathcal{K} \perp$ $P_{\mathcal{K} \ominus \mathcal{H}} V_{i_{2} j_{2}} \mathcal{K}$ if $i_{1} \neq i_{2}, j_{1}=1,2, \ldots, n_{i_{1}}$, and $j_{2}=1,2, \ldots, n_{i_{2}}$. According to the definition of the Toeplitz kernel $K_{c}$, for any finitely supported sequence $\left\{h_{\omega}\right\}_{\omega \in \mathbf{F}_{n}^{+}} \subset \mathcal{H}$ we have

$$
\begin{aligned}
\sum_{\sigma, \omega \in \mathbf{F}_{n}^{+}}\left\langle K_{c}(\sigma, \omega) h_{\omega}, h_{\sigma}\right\rangle & =\sum_{\sigma, \omega \in \mathbf{F}_{n}^{+}}^{*}\left\langle T_{\alpha}^{*} T_{\beta} h_{\omega}, h_{\sigma}\right\rangle \\
& =\sum_{\sigma, \omega \in \mathbf{F}_{n}^{+}}^{*}\left\langle P_{\mathcal{H}} V_{\beta} h_{\omega}, P_{\mathcal{H}} V_{\alpha} h_{\sigma}\right\rangle \\
& =\sum_{\sigma, \omega \in \mathbf{F}_{n}^{+}}^{*}\left\langle\left(P_{\mathcal{H}}+P_{\mathcal{K} \ominus \mathcal{H}}\right) V_{\beta} h_{\omega},\left(P_{\mathcal{H}}+P_{\mathcal{K} \ominus \mathcal{H}}\right) V_{\alpha} h_{\sigma}\right\rangle \\
& =\sum_{\sigma, \omega \in \mathbf{F}_{n}^{+}}^{*}\left\langle V_{\beta} h_{\omega}, V_{\alpha} h_{\sigma}\right\rangle=\sum_{\sigma, \omega \in \mathbf{F}_{n}^{+}}^{*}\left\langle V_{\alpha}^{*} V_{\beta} h_{\omega}, h_{\sigma}\right\rangle \\
& =\sum_{\sigma, \omega \in \mathbf{F}_{n}^{+}}\left\langle V_{\sigma}^{*} V_{\omega} h_{\omega}, h_{\sigma}\right\rangle=\left\|\sum_{\sigma \in \mathbf{F}_{n}^{+}} V_{\sigma} h_{\sigma}\right\|^{2} \geq 0,
\end{aligned}
$$


where $\sum^{*}$ is taken over all $\sigma, \omega \in \mathbf{F}_{n}^{+}$such that $K_{c}(\sigma, \omega)=T_{\alpha}^{*} T_{\beta}$ (see the definition of $K_{c}$ ). This proves that the Toeplitz kernel $K_{c}$ is positive definite.

Conversely, assume that $K_{c}$ is positive definite. According to Theorem 5.1 , there exists a Hilbert space $\mathcal{K} \supset \mathcal{H}$ and $\left\{V_{\sigma}\right\}_{\sigma \in \mathbf{F}_{n}^{+}}$a semigroup of isometries on $\mathcal{K}$ such that

$$
K_{c}(\sigma, \omega)=\left.P_{\mathcal{H}} V_{\sigma}^{*} V_{\omega}\right|_{\mathcal{H}} \quad \text { for any } \sigma, \omega \in \mathbf{F}_{n}^{+} .
$$

Since the relation (5.2) holds, we infer that for each $i=1, \ldots, k$, the sequence of isometries $\left(V_{i 1}, \ldots, V_{i n_{i}}\right)$ is contractive (see [Po5]). Since

$$
T_{s_{i j}}=K_{c}\left(e, s_{i j}\right)=\left.P_{\mathcal{H}} V_{s_{i j}}\right|_{\mathcal{H}}
$$

for each $i=1, \ldots, k$, we have

$$
\sum_{j=1}^{n_{i}}\left\|T_{i j}^{*} h\right\|^{2} \leq \sum_{j=1}^{n_{i}}\left\|V_{i j}^{*} h\right\|^{2} \leq\|h\|^{2} \quad \text { for any } h \in \mathcal{H} .
$$

This shows that $\left(T_{i 1}, \ldots, T_{i n_{i}}\right)$ is a contractive sequence of operators for each $i=1,2, \ldots, k$. The proof is complete.

Let us remark that in the particular case when $k=1$ and $n_{1}=n$ we find again Corollary 2.2 from [Po5]. In the particular case when $n_{1}=\cdots=$ $n_{k}=1$ we obtain the following.

Corollary 5.3. Let $\left\{T_{1}, \ldots, T_{k}\right\} \subset B(\mathcal{H})$. Then $\left\{T_{1}, \ldots, T_{k}\right\}$ is a sequence of contractions if and only if the Toeplitz kernel

$$
K: \mathbf{F}_{k}^{+} \times \mathbf{F}_{k}^{+} \rightarrow B(\mathcal{H})
$$

defined by $K(\sigma, \omega)=K(\alpha, \beta)=T_{\alpha}^{*} T_{\beta}$, where $\sigma=\operatorname{gld}(\sigma, \omega) \alpha$ and $\omega=$ $\operatorname{gld}(\sigma, \omega) \beta$, is positive definite.

Let $\mathcal{C}_{\rho}(\rho>0)$ denote the set of all sequences $\left\{A_{i j}\right\}_{\substack{i=1,2, \ldots, k \\ j=1,2, \ldots, n_{i}}}$ of operators on a Hilbert space $\mathcal{H}$ for which there exists a sequence of isometries $\left\{V_{i j}\right\}_{\substack{i=1,2, \ldots, k \\ j=1,2, \ldots, n_{i}}}$ on a Hilbert space $\mathcal{K} \supset \mathcal{H}$ such that

$$
\sum_{i=1}^{n_{i}} V_{i j} V_{i j}^{*} \leq I_{\mathcal{H}}
$$

for each $i=1,2, \ldots, k$, and

$$
A_{i_{1} j_{1}} \cdots A_{i_{m} j_{m}}=\left.\rho P_{\mathcal{H}} V_{i_{1} j_{1}} \cdots V_{i_{m} j_{m}}\right|_{\mathcal{H}}
$$

for any $i_{q} \in\{1,2, \ldots, k\}, j_{q} \in\left\{1,2, \ldots, n_{i_{q}}\right\}, q \in\{1,2, \ldots, m\}$ and $m \geq 1$. Let $\mathcal{K}_{\rho}: \mathbf{F}_{n}^{+} \times \mathbf{F}_{n}^{+} \rightarrow B(\mathcal{H})$ be the Toeplitz kernel defined by $K_{\rho}(e, e)=I_{\mathcal{H}}$ and

$$
K_{\rho}(\sigma, \omega)=\frac{1}{\rho} K_{c}(\sigma, \omega)
$$


if $\sigma \in \mathbf{F}_{n}^{+} \backslash\{e\}$ or $\omega \in \mathbf{F}_{n}^{+} \backslash\{e\}$, where $K_{c}$ is the Toeplitz kernel associated to $\left\{A_{i j}\right\}_{\substack{i=1,2, \ldots, k \\ j=1,2, \ldots, n_{i}}}$ (see the definition following Theorem 5.1).

Applying Theorem 5.2, we infer the following.

Theorem 5.4. $\left\{A_{i j}\right\}_{\substack{i=1,2, \ldots, k \\ j=1,2, \ldots, n_{i}}} \in \mathcal{C}_{\rho}$ if and only if the Toeplitz kernel $\mathcal{K}_{\rho}$ is positive definite.

One can prove that the class $\mathcal{C}_{\rho}(0<\rho<\infty)$ increases with $\rho$, i.e., $\mathcal{C}_{\rho} \subset \mathcal{C}_{\rho^{\prime}}$ and $\mathcal{C}_{\rho} \neq \mathcal{C}_{\rho}^{\prime}$ for $0<\rho<\rho^{\prime}<\infty$ (see [Po5] for a particular case).

The von Neumann inequality (3.7) can be extended, in an apropriate form, to the class $\mathcal{C}_{\rho}$.

Theorem 5.5. If $\left\{A_{i j}\right\}_{\substack{i=1,2, \ldots, k \\ j=1,2, \ldots, n_{i}}} \in \mathcal{C}_{\rho}(\rho>0)$, then for any polynomial $p\left(e,\left\{x_{i j}\right\}\right) \in O A_{+}\left(\Delta_{x y}, \mathcal{R}_{x y}\right)$,

$$
\left\|p\left(I,\left\{A_{i j}\right\}\right)\right\| \leq\left\|(1-\rho) p(e,\{0\})+\rho p\left(e,\left\{x_{i j}\right\}\right)\right\|_{O A_{+}\left(\Delta_{x y}, \mathcal{R}_{x y}\right)} .
$$

Corollary 5.6. Let $p\left(\left\{x_{i j}\right\}\right) \in O A_{+}\left(\Delta_{x y}, \mathcal{R}_{x y}\right)$ be such that $p(\{0\})=0$ and

$$
\left\|p\left(\left\{x_{i j}\right\}\right)\right\|_{O A_{+}\left(\Delta_{x y}, \mathcal{R}_{x y}\right)} \leq 1 .
$$

If $\left\{A_{i j}\right\}_{\substack{i=1,2, \ldots, k \\ j=1,2, \ldots, n_{i}}} \in \mathcal{C}_{\rho}(\rho>0)$ then $p\left(\left\{A_{i j}\right\}\right) \in \mathcal{C}_{\rho}$ (in the classical sense).

A sequence of operators $\left\{A_{i j}\right\}_{\substack{i=1,2, \ldots, k \\ j=1,2, \ldots, n_{i}}}$ is called simultaneously similar to a sequence $\left\{T_{i j}\right\}_{\substack{i=1,2, \ldots, k \\ j=1,2, \ldots, n_{i}}}$ if there is an invertible operator $X$ such that $A_{i j}=X T_{i j} X^{-1}$ for any $i=1,2, \ldots, k, j=1,2, \ldots, n_{i}$.

In what follows we consider an extension of the result of Sz.-Nagy and Foiaş [SzF1] and also [Po5].

Theorem 5.7. Any sequence $\left\{A_{i j}\right\}_{\substack{i=1,2, \ldots, k \\ j=1,2, \ldots, n_{i}}} \in \mathcal{C}_{\rho}(\rho>0)$ is simultaneously similar to a sequence $\left\{T_{i j}\right\}_{\substack{i=1,2, \ldots, k \\ j=1,2, \ldots, n_{i}}} \in \mathcal{C}_{1}$.

Proof. The inequality (5.3) can be extended to matrices. One can easily prove that, for any polynomials $p_{r s}\left(e,\left\{x_{i j}\right\}\right), 1 \leq r, s \leq m$,

$$
\left\|\left[p_{r s}\left(I,\left\{A_{i j}\right\}\right)\right]\right\| \leq(|1-\rho|+\rho)\left\|\left[p_{r s}\left(e,\left\{x_{i j}\right\}\right)\right]\right\|_{\Delta_{x y}} .
$$

This shows that the map $\Phi: \mathcal{P}_{x} \rightarrow B(\mathcal{H})$ defined by

$$
\Phi\left(p\left(e,\left\{x_{i j}\right\}\right)\right)=p\left(I,\left\{A_{i j}\right\}\right)
$$

can be extended to a completely bounded homomorphism of the free product disc algebra $O A_{+}\left(\Delta_{x y}, \mathcal{R}_{x y}\right)$. Now, according to Theorem 3.9, the result follows.

In the particular case when $k=1, n_{1}=1$ we find again the classical result of Sz.-Nagy and Foiaş [SzF1]. 


\section{Trigonometric moment problem for some free product $C^{*}$-algebras.}

As in the previous section, let $\mathbf{F}_{n_{i}}^{+} \quad(i=1,2, \ldots, k)$ be the unital free semigroup on $n_{i}$ generators: $g_{i 1}, g_{i 2}, \ldots, g_{i n_{i}}$, and let $e$ be the neutral element. Then $\Lambda:=\mathbf{F}_{n_{1}}^{+} * \cdots * \mathbf{F}_{n_{k}}^{+}$is the unital free semigroup on $n=n_{1}+\cdots+n_{k}$ generators.

For each $i=1,2, \ldots, k$, let $\left\{x_{i j}\right\}_{\substack{i=1,2, \ldots, k \\ j=1,2, \ldots, n_{i}}}$ and $\left\{y_{i j}\right\}_{\substack{i=1,2, \ldots, k \\ j=1,2, \ldots, n_{i}}}$ be ordinary variables satisfying the relation

$$
y_{i \alpha} x_{i \beta}=\delta_{\alpha, \beta} e
$$

for any $i=1,2, \ldots, k$, and $\alpha, \beta=1,2, \ldots, n_{i}$. For each $\sigma=g_{i_{1} j_{1}} \cdots g_{i_{p} j_{p}} \in \Lambda$ let $\tilde{\sigma}:=g_{i_{p} j_{p}} \cdots g_{i_{1} j_{1}}, x_{\sigma}=x_{i_{1} j_{1}} \cdots x_{i_{p} j_{p}}$, and $y_{\sigma}=y_{i_{1} j_{1}} \cdots y_{i_{p} j_{p}}$. If $\sigma=e$ (the neutral element in $\Lambda$ ) then we set $x_{e}=y_{e}:=e$ (the neutral element in $\Delta_{x y}$ (see Section 3)).

If $\pi$ is a representation of the universal algebra $O A\left(\Lambda_{x y}, \mathcal{R}_{x y}\right)$ on $B(\mathcal{K})$ then, according to Theorem 3.1, it is determined by contractive sequences of isometries $\left(S_{i 1}, \ldots, S_{i n_{i}}\right) \quad(i=1,2, \ldots, k)$ on the same Hilbert space $\mathcal{K}$ such that $\pi\left(x_{i j}\right)=S_{i j}, \pi\left(y_{i j}\right)=S_{i j}^{*}$ and $\pi(e)=I_{\mathcal{K}}$. Notice that for each $\sigma \in \Lambda$ we have $\pi\left(x_{\sigma}\right)=S_{\sigma}, \pi\left(y_{\tilde{\sigma}}\right)=S_{\sigma}^{*}=\pi\left(y_{\sigma}\right)^{*}$. According to the relation (6.1), $y_{\tilde{\sigma}} x_{\omega} \quad(\sigma, \omega \in \Lambda \backslash\{e\})$ is a reduced word if and only if there exist $i_{1}, i_{2} \in\{1,2, \ldots, k\}, i_{1} \neq i_{2}$, such that $\omega$ (resp. $\sigma$ ) starts, in its unique representation, with a generator of $\mathbf{F}_{n_{i_{1}}}^{+}\left(\operatorname{resp} . \mathbf{F}_{n_{i_{2}}}^{+}\right)$.

Define the following subsets of $\Lambda \times \Lambda$ :

$$
\begin{aligned}
\Gamma_{1} & =\{(e, \sigma): \sigma \in \Lambda\} \\
\Gamma_{2} & =\{(\omega, e): \omega \in \Lambda\} ; \\
\Gamma_{3} & =\left\{(\omega, \sigma): \omega, \sigma \in \Lambda \backslash\{e\} \text { and } y_{\tilde{\sigma}} x_{\omega} \text { is a reduced word }\right\} ; \\
\Gamma & =\Gamma_{1} \cup \Gamma_{2} \cup \Gamma_{3} .
\end{aligned}
$$

Notice that if $(\omega, \sigma) \in \Gamma$ then $(\sigma, \omega) \in \Gamma$. On the other hand, if $k=1$, then $\Gamma=\Gamma_{1} \cup \Gamma_{2}$. Let $\left\{A_{(\sigma, \omega)}\right\}_{(\sigma, \omega) \in \Gamma}$ be a sequence of operators in $B(\mathcal{H})$ such that $A_{(\sigma, \omega)}=A_{(\omega, \sigma)}^{*}$ for any $(\sigma, \omega) \in \Gamma$, and $A_{(e, e)}=I_{\mathcal{H}}$.

For any $\sigma, \omega \in \Lambda$ let us denote by $\operatorname{gld}(\sigma, \omega)$ the greatest left common divisor of them. Therefore,

$$
\sigma=\operatorname{gld}(\sigma, \omega) \alpha \quad \text { and } \quad \omega=\operatorname{gld}(\sigma, \omega) \beta
$$

for some $\alpha, \beta \in \Lambda$ with $\operatorname{gld}(\alpha, \beta)=e$. We associate to the sequence of operators $\left\{A_{(\sigma, \omega)}\right\}_{(\sigma, \omega) \in \Gamma}$ the kernel $K_{A}: \Lambda \times \Lambda \rightarrow B(\mathcal{H})$ defined by

$$
K_{A}(\sigma, \omega):= \begin{cases}A_{(\alpha, \beta)} & \text { if }(\alpha, \beta) \in \Gamma \\ 0 & \text { if }(\alpha, \beta) \notin \Gamma .\end{cases}
$$


It is easy to see that $K_{A}(e, e)=I_{\mathcal{H}}$ and $K_{A}(\alpha \sigma, \alpha \omega)=K_{A}(\sigma, \omega)$ for any $\alpha, \sigma, \omega \in \Lambda$, i.e., $K_{A}$ is a Toeplitz kernel. Notice that if $i=1,2, \ldots, k ; j_{1}, j_{2} \in$ $\left\{1,2, \ldots, n_{i}\right\}$ with $j_{1} \neq j_{2}$ then

$$
K_{A}\left(g_{i j_{1}} \sigma, g_{i j_{2}} \omega\right)=0
$$

for any $\sigma, \omega \in \Lambda$. Define the operator matrix

$$
M_{m}=\left[K_{A}(\sigma, \omega)\right]_{|\sigma| \leq m,|\omega| \leq m},
$$

where $|\sigma|$ stands for the length of $\sigma \in \mathbf{F}_{n}^{+}$. Denote

$$
\Gamma_{m}:=\{(\alpha, \beta) \in \Gamma:|\alpha| \leq m,|\beta| \leq m\}, m=1,2, \ldots
$$

In what follows we extend the operatorial trigonometric moment problem $[\mathbf{A k}]$ (see also $[\mathbf{P o 5}])$ to the free product $C^{*}$-algebra ${ }^{*} \mathbf{C} \mathcal{T}_{n_{i}}$.

Theorem 6.1. Let $\left\{A_{(\alpha, \beta)}\right\}_{(\alpha, \beta) \in \Gamma_{m}}$ be a sequence of operators in $B(\mathcal{H})$ such that $A_{(e, e)}=I_{\mathcal{H}}$ and $A_{(\alpha, \beta)}=A_{(\beta, \alpha)}^{*}$ for any $(\alpha, \beta) \in \Gamma_{m}$. If

$$
\mu: O A\left(\Lambda_{x y}, \mathcal{R}_{x y}\right) \rightarrow B(\mathcal{H})
$$

is a completely positive linear map such that

$$
\mu\left(y_{\tilde{\alpha}} x_{\beta}\right)=A_{(\alpha, \beta)}
$$

for any $(\alpha, \beta) \in \Gamma_{m}$, then $M_{m}$ is positive.

Conversely, if $M_{m}$ is positive then there is a completely positive linear map $\mu: O A\left(\Lambda_{x y}, \mathcal{R}_{x y}\right) \rightarrow B(\mathcal{H})$ such that $\mu\left(y_{\tilde{\alpha}} x_{\beta}\right)=A_{(\alpha, \beta)}$ for any $(\alpha, \beta) \in$ $\Gamma_{m-1}$.

Proof. Assume that $\mu: O A\left(\Lambda_{x y}, \mathcal{R}_{x y}\right) \rightarrow B(\mathcal{H})$ is a completely positive linear map such that

$$
\mu\left(y_{\tilde{\alpha}} x_{\beta}\right)=A_{(\alpha, \beta)} \quad \text { for any }(\alpha, \beta) \in \Gamma_{m} .
$$

According to Stinespring's theorem $[\mathbf{S}]$, there is a Hilbert space $\mathcal{K} \supset \mathcal{H}$ and a representation $\pi: O A\left(\Lambda_{x y}, \mathcal{R}_{x y}\right) \rightarrow B(\mathcal{H})$ such that

$$
\mu(f)=\left.P_{\mathcal{H}} \pi(f)\right|_{\mathcal{H}}, \quad f \in O A\left(\Lambda_{x y}, \mathcal{R}_{x y}\right) .
$$

Let $K: \Lambda \times \Lambda \rightarrow B(\mathcal{H})$ be the kernel defined by $K(\sigma, \omega)=\left.P_{\mathcal{H}} \pi\left(y_{\tilde{\sigma}} x_{\omega}\right)\right|_{\mathcal{H}}$ for any $\sigma, \omega \in \Lambda$. It is easy to see that $K(e, e)=I_{\mathcal{H}}, K(\omega, \sigma)=K(\sigma, \omega)^{*}$ and $K(\alpha \sigma, \alpha \omega)=K(\sigma, \omega)$ for any $\alpha, \sigma, \omega \in \Lambda$. Since for any finitely supported sequence $\left\{h_{\omega}\right\}_{\omega \in \Lambda} \subset \mathcal{H}$,

$$
\begin{aligned}
\sum_{\sigma, \omega \in \Lambda}\left\langle K(\sigma, \omega) h_{\omega}, h_{\sigma}\right\rangle & =\sum_{\sigma, \omega \in \Lambda}\left\langle P_{\mathcal{H}} \pi\left(y_{\tilde{\sigma}} x_{\omega}\right) h_{\omega}, h_{\sigma}\right\rangle \\
& =\sum_{\sigma, \omega \in \Lambda}\left\langle\pi\left(x_{\omega}\right) h_{\omega}, \pi\left(y_{\sigma}\right) h_{\sigma}\right\rangle=\sum_{\omega \in \Lambda}\left\|\pi\left(x_{\omega}\right) h_{\omega}\right\|^{2} \geq 0,
\end{aligned}
$$

we infer that $K$ is a positive definite Toeplitz kernel. 
In particular, the matrix $[K(\sigma, \omega)]_{|\sigma| \leq m,|\omega| \leq m}$ is positive. According to (6.4) and (6.5), it is a routine to show that $K(\sigma, \omega)=K_{A}(\sigma, \omega)$ for any $\sigma, \omega \in \Gamma$ with $|\sigma| \leq m,|\omega| \leq m$. Therefore, the matrix $M_{m}$ is positive.

Conversely, assume that the matrix $M_{m}$ is positive. Let $\mathcal{K}_{m}^{0}$ be the Hilbert space of all sequences of the form $\left\{h_{\sigma}\right\}_{\substack{\sigma \in \Lambda \\|\sigma| \leq m}}\left(h_{\sigma} \in \mathcal{H}\right)$ with the bilinear form $\langle\cdot, \cdot\rangle$ on $\mathcal{K}_{m}^{0}$ defined by

$$
\left\langle\left\{h_{\omega}\right\}_{|\omega| \leq m},\left\{h_{\sigma}^{\prime}\right\}_{|\sigma| \leq m}\right\rangle=\sum_{\substack{\omega, \sigma \in \Lambda \\|\omega|,|\sigma| \leq m}}\left\langle K_{A}(\sigma, \omega) h_{\omega}, h_{\sigma}^{\prime}\right\rangle_{\mathcal{H}} .
$$

Since $M_{m}$ is positive $\langle\cdot, \cdot\rangle$ is positive semi-definite. Consider

$$
\mathcal{N}_{m}=\left\{k \in \mathcal{K}_{m}^{0}:\langle k, k\rangle=0\right\}
$$

and $\mathcal{K}_{m}^{0} / \mathcal{N}_{m}$. Let $\mathcal{K}_{m}$ be the Hilbert space obtained by completing $\mathcal{K}_{m}^{0} / \mathcal{N}_{m}$ with the induced inner product.

Let $\mathcal{X}^{0}$ be the subspace of $\mathcal{K}_{m}^{0}$ defined by

$$
\mathcal{X}^{0}=\left\{\left\{h_{\sigma}\right\} \in \mathcal{K}_{m}^{0}: h_{\sigma}=0 \text { for }|\sigma|=m\right\}
$$

and let $\mathcal{X}=\mathcal{X}^{0} / \mathcal{N}_{m} \subset \mathcal{K}_{m}$. For each generator $g_{i j}(i=1,2, \ldots, k ; j=$ $\left.1,2, \ldots, n_{i}\right)$ of $\Lambda$ let $V_{i j}: \mathcal{X} \rightarrow \mathcal{K}_{m}$ be defined by

$$
V_{i j}\left(\left\{h_{\sigma}\right\}\right)=\left\{\delta_{g_{i j} \sigma}(t) h_{\sigma}\right\}_{|t| \leq m} .
$$

Define also $T_{i j}: \mathcal{X} \rightarrow \mathcal{X}$ by $T_{i j}^{*}=V_{i j}^{*} \mid \mathcal{X}$. Embed $\mathcal{H}$ in $\mathcal{K}_{m}$ by setting $h=\left\{\delta_{e}(t) h\right\}_{|t| \leq m}$. This identification is allowed since it preserves the linear and metric structure of $\mathcal{H}$.

For any $(\sigma, \omega) \in \Gamma_{m-1}$ and $h, h^{\prime} \in \mathcal{H}$ we have

$$
\begin{aligned}
\left\langle P_{\mathcal{H}} T_{\sigma}^{*} T_{\omega} h, h^{\prime}\right\rangle_{\mathcal{H}} & =\left\langle T_{\omega} h, T_{\sigma} h^{\prime}\right\rangle_{\mathcal{K}_{m}} \\
& =\left\langle P_{\mathcal{X}}\left(\left\{\delta_{\omega}(t) h\right\}_{|t| \leq m}\right), P \mathcal{X}\left(\left\{\delta_{\sigma}(s) h^{\prime}\right\}_{|s| \leq m}\right)\right\rangle_{\mathcal{K}_{m}} \\
& =\left\langle\left\{\delta_{\omega}(t) h\right\}_{|t| \leq m},\left\{\delta_{\sigma}(s) h^{\prime}\right\}_{|s| \leq m}\right\rangle_{\mathcal{K}_{m}} \\
& =\sum_{\substack{s, t \in \Lambda \\
|s|,|t| \leq m}}\left\langle K_{A}(s, t) \delta_{\omega}(t) h, \delta_{\sigma}(s) h^{\prime}\right\rangle_{\mathcal{H}} \\
& =\left\langle K_{A}(\sigma, \omega) h, h^{\prime}\right\rangle_{\mathcal{H}} .
\end{aligned}
$$

Therefore, $K_{A}(\sigma, \omega)=\left.P_{\mathcal{H}} T_{\sigma}^{*} T_{\omega}\right|_{\mathcal{H}}$ for any $(\sigma, \omega) \in \Gamma_{m-1}$. Let us remark that this relation holds, in fact, for any $(\sigma, \omega) \in \Gamma_{m}$ such that either $|\sigma| \leq m-1$ or $|\omega| \leq m-1$.

Let us show that for each $i=1,2, \ldots, k$,

$$
T_{i 1} T_{i 1}^{*}+\cdots+T_{i n_{i}} T_{i n_{i}}^{*} \leq I_{\mathcal{X}}
$$

Since $\sum_{j=1}^{n_{i}} T_{i j} T_{i j}^{*}=\left.P_{\mathcal{X}} \sum_{j=1}^{n_{i}} V_{i j} V_{i j}^{*}\right|_{\mathcal{X}}$, it is enough to prove that $\left(V_{i 1}, \ldots, V_{i n_{i}}\right)$ is a sequence of isometries with orthogonal ranges. According to (6.6) and 
(6.2), for $j_{1} \neq j_{2} ; j_{1}, j_{2} \in\left\{1,2, \ldots, n_{i}\right\}$, we have

$$
\begin{aligned}
& \left\langle V_{i j_{1}}\left\{h_{\omega}\right\}, V_{i j_{2}}\left\{h_{\sigma}^{\prime}\right\}\right\rangle_{\mathcal{K}_{m}} \\
& =\sum_{|\sigma|,|\omega| \leq m-1}\left\langle K_{A}\left(g_{i j_{2}} \sigma, g_{i j_{1}} \omega\right) h_{\omega}, h_{\sigma}^{\prime}\right\rangle=0 .
\end{aligned}
$$

Since $K_{A}$ is a Toeplitz kernel one can similarly prove that $V_{i j}\left(j=1,2, \ldots, n_{i}\right)$ are isometries. Therefore $\sum_{j=1}^{n_{i}} V_{i j} V_{i j}^{*} \leq I_{\mathcal{K}_{m}}$. Hence, and using the definition of $T_{i j}$ we infer the relation (6.7).

Let $\left\{W_{i j}\right\}_{\substack{i=1,2, \ldots, k \\ j=1,2, \ldots, n_{i}}}$ be the minimal isometric dilation of $\left\{T_{i j}\right\}_{\substack{i=1,2, \ldots, k \\ j=1,2, \ldots, n_{i}}}$ on a Hilbert space $\mathcal{K} \supset \mathcal{X}$ (see Theorem 2.1). Since

$$
P_{\mathcal{K} \ominus \mathcal{X}} W_{i_{1} j_{1}} \mathcal{K} \perp P_{\mathcal{K} \ominus \mathcal{X}} W_{i_{2} j_{2}}
$$

if $i_{1} \neq i_{2}, j_{1}=1,2, \ldots, n_{i_{1}}$ and $j_{2}=1,2, \ldots, n_{i_{2}}$, and $W_{i j_{1}} \mathcal{K} \perp W_{i j_{2}} \mathcal{K}$ for any $i=1,2, \ldots, k$ and $j_{1}, j_{2} \in\left\{1,2, \ldots, n_{i}\right\}$ such that $j_{1} \neq j_{2}$, a simple computation shows that $T_{\sigma}^{*} T_{\omega}=P_{\mathcal{X}} W_{\sigma}^{*} W_{\omega} \mid \mathcal{X}$, for any $(\sigma, \omega) \in \Gamma_{m}$. Therefore, for any $(\sigma, \omega) \in \Gamma_{m-1}$ we have

$$
K_{A}(\sigma, \omega)=\left.P_{\mathcal{H}} T_{\sigma}^{*} T_{\omega}\right|_{\mathcal{H}}=\left.P_{\mathcal{H}} W_{\sigma}^{*} W_{\omega}\right|_{\mathcal{H}} .
$$

Define $\mu: O A\left(\Lambda_{x y}, R_{x y}\right) \rightarrow B(\mathcal{H})$ by

$$
\mu(f)=\left.P_{\mathcal{H}} \pi(f)\right|_{\mathcal{H}}
$$

where $\pi: O A\left(\Lambda_{x y}, \mathcal{R}_{x y}\right) \rightarrow B(\mathcal{K})$ is a representation determined by $\pi(e)=$ $I_{\mathcal{K}}, \pi\left(x_{i j}\right)=W_{i j}$ and $\pi\left(y_{i j}\right)=W_{i j}^{*}$. Thus, $\mu$ is a completely positive linear map. On the other hand, using the relations (6.8) and (6.9), we infer that

$$
\mu\left(y_{\tilde{\alpha}} x_{\beta}\right)=\left.P_{\mathcal{H}} W_{\alpha}^{*} W_{\beta}\right|_{\mathcal{H}}=K_{A}(\alpha, \beta)=A_{(\alpha, \beta)}
$$

for any $(\alpha, \beta) \in \Gamma_{m-1}$, which completes the proof.

Notice that if $k=1$ then the relation (6.10) is true for any $(\alpha, \beta) \in \Gamma_{m}$. Let us remark that in the particular case when $k=1$ and $n_{1}=n$ we have $\Gamma_{3}=\emptyset, \Gamma=\Gamma_{1} \cup \Gamma_{2}$ and $\Lambda=\mathbf{F}_{n}^{+}$, and we find again Theorem 3.1 from [Po5].

Corollary 6.2. Let $\left\{A_{(\sigma, \omega)}\right\}_{(\sigma, \omega) \in \Gamma}$ be a sequence of operators in $B(\mathcal{H})$ such that $A_{(\sigma, \omega)}=A_{(\omega . \sigma)}^{*}$ for any $(\sigma, \omega) \in \Gamma$ and $A_{(e, e)}=I_{\mathcal{H}}$.

Then, there is a completely positive linear map $\mu: O A\left(\Lambda_{x y}, \mathcal{R}_{x y}\right) \rightarrow B(\mathcal{H})$ such that $\mu\left(y_{\tilde{\alpha}} x_{\beta}\right)=A_{(\alpha, \beta)}$ for any $(\alpha, \beta) \in \Gamma$ if and only if the Toeplitz kernel $K_{A}$ is positive definite.

Let us recall that $O A\left(\Delta_{x^{\prime} y^{\prime}}, \mathcal{R}_{x^{\prime} y^{\prime}}\right)$ is completely isometrically isomorphic to the free product $C^{*}$-algebra $\check{*}_{\mathbf{C}} \mathcal{O}_{n_{i}}$.

Using Theorem 2.5 and Arverson's extension theorem [Ar], one can easily adapt the last part of the proof of Theorem 6.1 to obtain the following results. 
Corollary 6.3. If the operator matrix $M_{m}$ is positive definite, then there is a completely positive map

$$
\psi: O A\left(\Delta_{x^{\prime} y^{\prime}}, \mathcal{R}_{x^{\prime} y^{\prime}}\right) \rightarrow B(\mathcal{H})
$$

such that $\psi\left(x_{\sigma}\right)=A_{(e, \sigma)}$ for any $\sigma \in \Lambda,|\sigma| \leq m$.

Notice that in the particular case when $k=1$ the converse of the above corollary also holds. Therefore, in the particular case when $k=1, n_{1}=n$ we have $\Lambda=\mathbf{F}_{n}^{+}$and we infer the following trigonometric moment problem for the Cuntz algebra $\mathcal{O}_{n}$.

Let $v_{1}, v_{2}, \ldots, v_{n}$ be a system of generators of the Cuntz algebra $\mathcal{O}_{n}$. Let $\mathbf{F}_{n}^{+}$be the unital free semigroup on $n$ generators: $g_{1}, g_{2}, \ldots, g_{n}$. For each $\sigma=g_{i_{1}} \cdots g_{i_{p}} \in \mathbf{F}_{n}^{+}$denote $v_{\sigma}=v_{i_{1}} \cdots v_{i_{p}}$ and $v_{e}=1$.

Corollary 6.4. Let $\left\{B_{(\sigma)}\right\}_{\sigma \in \mathbf{F}_{n}^{+}}$be a sequence of operators in $B(\mathcal{H})$ with $B_{(e)}=I_{\mathcal{H}}$. Then, there is a completely positive linear map $\mu: \mathcal{O}_{n} \rightarrow$ $B(\mathcal{H})$ such that $\mu\left(v_{\sigma}\right)=B_{(\sigma)}, \sigma \in \mathbf{F}_{n}^{+}$, if and only if the Toeplitz kernel $K: \mathbf{F}_{n}^{+} \times \mathbf{F}_{n}^{+} \rightarrow B(\mathcal{H})$ defined by $K(e, e)=I_{\mathcal{H}}$ and

$$
K(\sigma, \omega)= \begin{cases}B_{(\tau)}^{*} & \text { if } \sigma=\omega \tau \text { for some } \tau \in \mathbf{F}_{n}^{+} \\ B_{(\tau)} & \text { if } \omega=\sigma \tau \text { for some } \tau \in \mathbf{F}_{n}^{+} \\ 0 & \text { otherwise }\end{cases}
$$

is positive definite.

In the particular case when $n=1$ we find again the classical operatorial trigonometric moment problem $[\mathbf{A k}]$.

Corollary 6.5. Given the operators $A_{k} \in B(\mathcal{H}), k=0,1, \ldots, m \quad\left(A_{0}=I\right)$, there exists a positive linear map $\mu: C(\mathbf{T}) \rightarrow B(\mathcal{H})$ such that $\mu\left(e^{i k t}\right)=A_{k}$, $k=0,1, \ldots, m$, if and only if the block matrix

$$
\left[\begin{array}{cccc}
I_{\mathcal{H}} & A_{1} & \cdots & A_{m} \\
A_{1}^{*} & I_{\mathcal{H}} & \cdots & A_{m-1} \\
\vdots & \vdots & & \vdots \\
A_{m}^{*} & A_{m-1}^{*} & \cdots & I_{\mathcal{H}}
\end{array}\right]
$$

built up on the given operators $\left\{A_{k}\right\}_{k=1}^{m}$ is positive.

\section{References}

[A] J. Agler, The Arveson extension theorem and coanalytic models, Integral Equations Operator Theory, 5 (1982), 608-631.

[Ak] N.I. Akhiezer, The classical moment problem, Edimburg, Scotland: Olivier and Boyd, 1965.

[Ar] W.B. Arveson, Subalgebras of $C^{*}$-algebras, Acta. Math., 123 (1969), 141-224. 
[Av] D. Avitzour, Free products of $C^{*}$-algebras, Trans. Amer. Math. Soc., 271 (1982), 423-436.

[B] D.P. Blecher, Factorizations in universal operator spaces and algebras, Rocky Mountain J. Math., 27 (1997), 151-167.

[BP] D.P. Blecher and V. Paulsen, Explicit construction of universal operator algebras and applications to polynomial factorization, Proc. Amer. Math. Soc., 112 (1991), 839-850.

[Bo] F. Boca, Free products of completely positive maps and spectral sets, J. Funct. Anal., 97 (1991), 251-263.

[BD] F.F. Bonsall and J. Duncan, Complete normed algebras, Springer-Verlag, New York, Heidelberg, Berlin, 1973.

[Boz] M. Bozejko, Positive definite kernels, length functions on groups and noncommutative von Neumann inequality, Studia Math., 95 (1989), 107-118.

[BrJ] O. Bratteli and P.E.T. Jorgensen, Endomorphisms of $B(\mathcal{H})$, II: Finitely correlated states on $\mathcal{O}_{n}$, J. Funct. Anal., 145 (1997), 323-373.

[Bu] J.W. Bunce, Models for n-tuples of noncommuting operators, J. Funct. Anal., 57 (1984), 21-30.

[C] L.A. Coburn, The $C^{*}$-algebra generated by an isometry, Bull. Amer. Math. Soc., 13 (1967), 722-726.

[Cu] J. Cuntz, Simple $C^{*}$-algebras generated by isometries, Commun. Math. Phys., 57 (1977), 173-185.

[DSz] E. Durszt and B. Szokefalvi-Nagy, Remark to a paper of A.E. Frazho: "Models for noncommuting operators", J. Funct. Anal., 52 (1983), 146-147.

[E] D.E. Evans, On $O_{n}$, Publ. Res. Int. Math. Sci., 16 (1980), 915-927.

[F] A.E. Frazho, Complements to models for noncommuting operators, J. Funct. Anal., 59 (1984), 445-461.

[N] M.A. Naimark, Positive definite operator functions on a commutative group, Bulletin Acad. Sci. URSS, 7 (1943), 237-244.

[P1] V.I. Paulsen, Every completely polynomially bounded operator is similar to a contraction, J. Funct. Anal., 55 (1984), 1-17.

[P2] Completely Bounded Maps and Dilations, Pitman Research Notes in Mathematics, Vol. 146, New York, 1986.

[Pi] G. Pisier, Similarity Problems and Completely Bounded Maps, Springer Lect. Notes Math., 1618 (1995), Springer-Verlag, New York.

[Po1] G. Popescu, Isometric dilations for infinite sequences of noncommuting operators, Trans. Amer. Math. Soc., 316 (1989), 523-536.

[Po2] — Von Neumann inequality for $\left(B(H)^{n}\right)_{1}$, Math. Scand., 68 (1991), 292304.

[Po3] _ Functional calculus for noncommuting operators, Michigan Math. J., 42 (1995), 345-356.

[Po4] , Non-commutative disc algebras and their representations, Proc. Amer. Math. Soc., 124 (1996), 2137-2148.

[Po5] _ Positive-definite functions on free semigroups, Canadian J. Math., 48 (1996), 887-896. 
[Po6] _ Universal operator algebras associated to contractive sequences of noncommuting operators, J. London Math. Soc., to appear.

[Po7] - Poisson transforms on some $C^{*}$-algebras generated by isometries, J. Funct. Anal., to appear.

[Sc] J.J. Schäffer, On unitary dilations of contractions, Proc. Amer. Math. Soc., 127 (1955), 322.

[S] W.F. Stinespring, Positive functions on $C^{*}$-algebras, Proc. Amer. Math. Soc., 6 (1955), 211-216.

[SzF1] B.Sz.-Nagy and C. Foiaş, Similitude des operators de classe $C_{\rho}$ a des contractions, C. R. Acad. Sci. Paris, Serie A, 264 (1967), 1063-1065.

[SzF2] Harmonic analysis on operators on Hilbert space, North-Holland, Amsterdam, 1970.

[V] D. Voiculescu, Symmetries of some reduced free product $C^{*}$-algebras, LNM 1132, Operator Algebras and Their Connection to Topology and Ergodic Theory, Springer-Verlag, Berlin, (1985), 556-588.

[vN] J. von Neumann, Eine spectraltheorie für allgemeine operatoren eines unitären Raumes, Math. Nachr., 4 (1951), 258-281.

Received May 25, 1996 and revised February 1, 1998. This research was partially supported by NSF DMS-9531954.

The University of Texas at San Antonio

San Antonio, TX 78249

E-mail address: gpopescu@math.utsa.edu 